\title{
Effective Dynamics of a Magnetic Vortex in a Local potential
}

\author{
Y. Strauss and I.M. Sigal
}

Preprint 2003/2004, No. 13

\begin{abstract}
We study a gradient-flow version of the Ginzburg-Landau equations with an addition of a compactly supported potential term. We consider initial data close to a magnetic vortex solution of the Ginzburg-Landau equations and find the dynamical law governing the motion of the vortex center in the presence of the potential.
\end{abstract}

\section{Introduction}

\subsection{Preliminary discussion and statement of the problem}

In this work we consider the gradient flow Ginzburg-Landau equations in the presence of a local potential. Specifically, we study certain solutions of the system of equations

$$
\begin{aligned}
\partial_{t} \psi & =\Delta_{A} \psi+\frac{\lambda}{2}\left(1-|\psi|^{2}\right) \psi-\epsilon W \psi \\
\partial_{t} A & =-\nabla \times \nabla \times A+\operatorname{Im}\left(\bar{\psi} \nabla_{A} \psi\right)
\end{aligned}
$$

where $(\psi(t), A(t)): R^{2} \mapsto C \times R^{2}$ for each $t \geq 0, \nabla_{A}=\nabla-i A$ and $\Delta_{A}=\nabla_{A}^{2}$. The function $W: R^{2} \mapsto R$ is a localized potential and is assumed to be smooth and compactly supported. The parameter $\epsilon$ is the potential strength parameter.

Setting $\epsilon=0$ in Eq. (1.1), one obtains the following set of coupled equations for $\psi$ and $A$

$$
\begin{aligned}
\partial_{t} \psi & =\Delta_{A} \psi+\frac{\lambda}{2}\left(1-|\psi|^{2}\right) \psi \\
\partial_{t} A & =-\nabla \times \nabla \times A+\operatorname{Im}\left(\bar{\psi} \nabla_{A} \psi\right) .
\end{aligned}
$$

This system of equations is a particular dynamical model in the study of superconductivity known as the superconducting model, also called the Gorkov-Eliashberg equations or timedependent Ginzburg-Landau (TDGL) equations [GE,T]. In the study of superconductivity the function $\psi$ is the order parameter measuring the density of superconducting electrons and the vector field $A$ is the vector potential for the magnetic field.

The stationary solutions of the TDGL equations are solutions of the Ginzburg-Landau (GL) equations

$$
\begin{aligned}
\Delta_{A} \psi+\frac{\lambda}{2}\left(1-|\psi|^{2}\right) \psi & =0 \\
-\nabla \times \nabla \times A+\operatorname{Im}\left(\bar{\psi} \nabla_{A} \psi\right) & =0 .
\end{aligned}
$$


Denote by $\mathcal{E}_{G L}(u)$ the Ginzburg-Landau energy functional

$$
\mathcal{E}_{G L}(u)=\frac{1}{2} \int_{R^{2}}\left[\left|\nabla_{A} \psi\right|^{2}+|\nabla \times A|^{2}+\frac{\lambda}{4}\left(|\psi|^{2}-1\right)^{2}\right]
$$

where $u=(\psi, A)$. The Ginzburg-Landau equations, Eq. (1.3) are the Euler-Lagrange equations derived from $\mathcal{E}_{G L}$.

Superconductors are characterized by two length scales called penetration depth and coherence depth. In the scaling units corresponding to the form of $\mathcal{E}_{G L}$ given in Eq. (1.4) the penetration depth, measuring the scale of variations in the magnetic field, is equal to 1 . The coherence length, measuring the scale of variations in the order parameter $\psi$ is $1 / m_{\lambda}$ where $m_{\lambda}=\min (\sqrt{\lambda}, 2)$. For $\lambda=1$ the two length scales are equal and we distinguish between type I superconductors, for which $\lambda<1$ and type II superconductors for which $\lambda>1$. Experimentally, type I and type II materials differ in their magnetic behavior. In type I superconductors magnetic fields are excluded from the bulk of the material except for a very thin layer near the surface. In type II superconductors magnetic fields penetrate the material in vortex structures. In general, Type II superconductors can sustain magnetic fields much higher than type I superconductors without losing their superconducting state. The existence of magnetic vortices and of type II superconductors was predicted in 1957 by Abrikosov [A] (in addition Abrikosov predicted the existence of large arrays of magnetic vortices, called Abrikosov lattices, in type II materials).

Magnetic (Abrikosov) vortices are equivariant solutions of Eq. (1.3) of the form

$$
\psi^{(n)}(x)=f_{n}(r) e^{i n \theta}, \quad A^{(n)}(x)=a_{n}(r) \nabla(n \theta)
$$

where $(r, \theta)$ are the polar coordinates of $x \in R^{2}$ and $n \in Z$. In Eq. (1.4) configurations $(\psi, A)$ with finite energy are classified by the Brouwer degree of $\psi$, i.e., the topological degree

$$
\operatorname{deg}(\psi):=\operatorname{deg}\left(\left.\frac{\psi}{|\psi|}\right|_{|x|=R}\right), \quad \operatorname{deg}(\psi) \in Z
$$

where $R$ is large enough. For the magnetic field $B=\nabla \times A$ the degree map corresponds to the quantization of magnetic flux and we have

$$
\int_{R^{2}} B=2 \pi \operatorname{deg}(\psi) .
$$

Calculation of the degree of $\psi^{(n)}$ for a magnetic vortex solution given by Eq. (1.5) yields $\operatorname{deg}\left(\psi^{(n)}\right)=n$, so that magnetic vortices are characterized by the quantization of flux and the pair $\left(\psi^{(n)}, A^{(n)}\right)$ is called an $n$-vortex. Denoting $u=(\psi, A)$, we note that for an $n$-vortex $v^{(n)}=\left(\psi^{(n)}, A^{(n)}\right)$ the GL energy functional $\mathcal{E}_{G L}$ is a smooth functional on the affine space given by

$$
X^{(n)} \equiv\left\{u: R^{2} \rightarrow C \times R^{2} \mid u-v^{(n)} \in H^{1}\left(R^{2} ; C \times R^{2}\right)\right\} .
$$


In addition we note that $0<f_{n}<1,0<a_{n}<1$ and that the asymptotic behavior for $a_{n}(r)$ and $f_{n}(r)$ as $r \rightarrow \infty$ is known to be given by (see $[\mathrm{BC}]$ ):

$$
f_{n}(r)=1+O\left(e^{-m_{\lambda} r}\right), \quad a_{n}(r)=1+O\left(e^{-r}\right) .
$$

It is also known that $f_{n}(r)$ vanishes like $r^{n}$ and $a_{n}(r)$ vanishes like $r^{2}$ as $r$ goes to the origin.

The existence of $n$-vortices was proved by Pholr $[\mathrm{P}]$ and by Berger and Chen $[\mathrm{BC}]$ by variational methods. Stability of $n$-vortices was proved by Gustafson and Sigal [GS1].

As mentioned above, type II superconductors can sustain very large magnetic fields (over $10^{5}$ Gauss). However, a major obstacle in the attempt to produce large magnetic fields is the dissipation of energy due to the creeping or flow of vortices [T]. One way to overcome this problem is to pin down the vortices to particular locations in the material. The pinning down of vortices is achieved by the presence of point defects, impurities, inhomogeneities or by a variation in the thickness of the sample of superconducting material [DG]. Rubinstein [Rub1] considered a model of the effect of pinning in which the quartic term in $\mathcal{E}_{G L}$ is replaced by the modified term

$$
V_{f}(\psi ; x)=\frac{\lambda}{2}\left(|\psi|^{2}-f^{2}(x)\right)^{2} .
$$

The function $f(x)$ can be thought of as measuring the quality of the superconductor (consider for example the large $\lambda$ limit). In the model analyzed by Rubinstein the superconductor occupies a bounded domain $\Omega$ and the function $f(x)$ is taken to be

$$
f(x)=1+\left(a_{1}-1\right) \chi\left(\Omega_{1}\right)
$$

where $\chi\left(\Omega_{1}\right)$ is the characteristic function of a region $\Omega_{1}$, which is taken to be in the interior of $\Omega$, and $a_{1}<1$. It was proved in [Rub1] that if a configuration $\left(\psi_{1}, A_{1}\right)$ contains a vortex in $\Omega_{2}=\Omega-\Omega_{1}$, then, for a value of $\lambda$ large enough, there exists another configuration $\left(\psi_{2}, A_{2}\right)$ containing a vortex in $\Omega_{1}$ such that $\mathcal{E}_{G L}\left(\psi_{2}, A_{2}\right)<\mathcal{E}_{G L}\left(\psi_{1}, A_{1}\right)$.

In $[\mathrm{BBH}]$ Bethuel, Brezis and Hélein study the large $\lambda$ limit of a simplified version of the GL functional. Assuming that $\Omega$ is a simply connected bounded domain in $\mathrm{C}$, Bethuel, Brezis and Hélein consider the functional

$$
\mathcal{E}_{\epsilon}(\psi)=\int_{\Omega}\left[\frac{1}{2}|\nabla \psi|^{2}+\frac{1}{4 \epsilon^{2}}\left(|\psi|^{2}-1\right)^{2}\right] .
$$

Since there is no magnetic field in the problem one needs to introduce a mechanism for the confinment of vortices to $\Omega$. The minimization problem for $\mathcal{E}_{\epsilon}(\psi)$ is considered, therefore, over all $\psi$ satisfying the Dirichlet boundary condition

$$
\psi(x)=g(x), \quad x \in \partial \Omega
$$

where $g: \partial \Omega \rightarrow S^{1}$ satisfies $\operatorname{deg} g=d \neq 0$. It was proved in $[\mathrm{BBH}]$ that, if $\Omega$ is a star shaped domain in $\mathrm{C}$, there are $N$ points $a_{1}, \ldots, a_{N}, N$ integers $d_{1}, \ldots, d_{N}$ and a sequence $\psi_{\epsilon_{n}}$ of critical points of $\mathcal{E}_{\epsilon}(\psi)$, corresponding to a sequence $\epsilon_{n} \rightarrow 0$, such that $\psi_{\epsilon_{n}} \rightarrow u_{*}$ in $C_{\text {loc }}^{k, \alpha}\left(\Omega \backslash\left\{a_{1}, \ldots, a_{N}\right\}, C\right)$ where $u_{*}: \Omega \backslash\left\{a_{1}, \ldots, a_{N}\right\} \rightarrow S^{1}$ is an harmonic map defined by 


$$
u_{*}=e^{i \Phi} \prod_{j=1}^{N}\left(\frac{z-a_{j}}{\left|z-a_{j}\right|}\right)^{d_{j}}
$$

and where the harmonic function $\Phi$ is determined by the condition $u_{*}=g$ on $\partial \Omega$. Furthermore, if $\psi_{\epsilon_{n}}$ is a sequence of minimizers we have $d_{j}=+1$ for all $j=1, \ldots, N=d$ and the set of distinct points $a_{1}, \ldots, a_{d}$ is a global minimum of a renormalized energy functional $\mathcal{E}_{g}$ involving $d$ points and the harmonic Green function of $\Omega[\mathrm{BR}, \mathrm{S}]$ (see also [PaRi]). In the general context of the Bethuel-Brezis-Hélein theory Andre and Shafrir [AS] analyzed a model for the pinning of vortices in superconductors with small variable thickness which was proposed by Du and Gunzburger [DG]. This model for the pinning effect involves a weighted GL functional of the form

$$
\mathcal{E}_{\epsilon}(\psi)=\int_{\Omega} p(x)\left[\frac{1}{2}|\nabla \psi|^{2}+\frac{1}{4 \epsilon^{2}}\left(|\psi|^{2}-1\right)^{2}\right] .
$$

For this model Andre and Shafrir proved the Bethuel-Brezis-Hélein type result that the set of points $a_{1}, \ldots, a_{d}$ defining $u_{*}$ consists of points (possibly repeating) in which $p$ achieves its global minimum.

More recently the effect of pinning was studied by I.M. Sigal and F. Ting [ST1] using methods closely related to those applied in the proof of the main theorem in the present paper. In addition, Sigal and Ting investigated the stability of pinned vortices [ST2]. Other results of recent work on the pinning of vortices can be found in Aftalion, Sandier and Serfaty [ASaSe] and Andre, Bauman and Philips [ABP].

Our goal in the present paper is to provide a description of the dynamics of the pinning effect, i.e., assuming that a magnetic vortex is not pinned down at an equlibrium position by a local pinning potenial, but is located at its near vicinity, we aim to give a description of the motion of the vortex under the influence of the potential. More specifically, we give an equation of motion for the vortex center and for the elecromagnetic gauge function in the presence of the potential. Formally speaking, this constitutes a reduction of the dynamics of the various fields in the problem to an effective dynamics of a smaller number of degrees of freedom, i.e, the vortex center and the gauge function. The methods utilized for the proof of the main theorem in the current work is essentially the same as those used by Gustafson and Sigal in their study of the dynamics of magnetic vortices [GS2]. In this context we mention that in [GS2] one can find a short survey of various rigorous and non-rigorous results concerning the dynamics of vortices in various time dependent versions of the GL equations. In particular, we mention here the non-rigorous results for the superconducting model of Eq. (1) obtained by Atiyah and Hitchin [AH], Perez and Rubinstein $[\mathrm{PeRu}]$ and $\mathrm{W} . \mathrm{E}[\mathrm{E}]$ and the rigorous results obtained, for the same equations, by Demoulini and Stuart [DS]. A simplified version of the TDGL equations, called the nonlinear heat flow (NLHF) equation, in which $A \equiv 0$, was studied by Neu [NEU], Lin [L], Jerard and Soner [JS] and Rubinstein and Sternberg [RS1] (see also [Rub2] and references therein).

We conclude this subsection by noting that reviews of the Ginzburg-Landau theory of superconductivity can be found, for example, in [BFGLV, Gu1, JT, Rit, Riv, Rub2]. 


\subsection{Symmetries of the Ginzburg-Landau equations}

The Ginzburg-Landau energy functional $\mathcal{E}_{G L}$ is invariant under the following symmetries:

(i) Translation symmery $x \rightarrow x^{\prime}=x+z$

$$
\psi(x) \rightarrow \psi_{z}(x)=\psi(x-z), \quad A(x) \rightarrow A_{z}(x)=A(x-z)
$$

(ii) Gauge symmetry

$$
\psi(x) \rightarrow \psi_{\gamma}(x)=e^{i \gamma(x)} \psi(x), \quad A(x) \rightarrow A_{\gamma}(x)=A(x)+\nabla \gamma(x) .
$$

(iii) Rotation Symmetry $x \rightarrow x^{\prime}=R x, R \in S O(2)$

$$
\psi(x) \rightarrow \psi_{R}(x)=\psi\left(R^{-1} x\right), \quad A(x) \rightarrow A_{R}(x)=R A\left(R^{-1} x\right) .
$$

We note that for an $n$-vortex solution the application of the rotation symmetry group correspond to a subgroup of the gauge symmetry. Therefore, for $n$-vortices the relevant symmetry operations are the translation and gauge groups. Accordingly, given an $n$ vortex $v^{(n)}=\left(\psi^{(n)}, A^{(n)}\right)$, we can apply translation symmetry with translation parameters $\mathbf{z}=\left(z_{1}, z_{2}\right) \in R^{2}$ and gauge symmetry with a gauge function $\gamma$ and find another solution $v_{\mathbf{z} \gamma}$ of Eq. (1.3) where

$$
v_{\mathbf{z} \gamma}=\left(\psi_{\mathbf{z} \gamma}, A_{\mathbf{z} \gamma}\right)=\left(e^{i \gamma} \psi^{(n)}(\cdot-\mathbf{z}), A^{(n)}(\cdot-\mathbf{z})+\nabla \gamma\right)=\left(e^{i \gamma} \psi_{\mathbf{z} 0}, A_{\mathbf{z} 0}+\nabla \gamma\right)
$$

and $\psi_{\mathbf{z} 0} \equiv \psi^{(n)}(\cdot-\mathbf{z})$ and $A_{\mathbf{z} 0} \equiv A^{(n)}(\cdot-\mathbf{z})$. For reasons that will become clear below we consider gauge transforms of the form

$$
\gamma=\sum_{i=1,2} z_{i} A_{i}^{(n)}(\cdot-\mathbf{z})+\tilde{\gamma}
$$

Then, according to Eq. (1.6) and Eq. (1.7) we require that $\tilde{\gamma} \in H^{2}\left(R^{2} ; R\right)$. In this case $v_{\mathbf{z} \gamma}$ in Eq. (1.7) will always be in $X^{(n)}$. Applying symmetry transformations in the form of Eq. (1.7) for all possible values of $\mathbf{z}$ and $\gamma$ we obtain the symmetry manifold of the $n$-vortex

$$
M_{\text {sym }}^{(n)}=\left\{v_{\mathbf{z} \gamma} \mid \mathbf{z} \in R^{2}, \tilde{\gamma} \in H^{2}\left(R^{2} ; R\right)\right\}
$$

with $\tilde{\gamma}$ given in Eq. (1.8). It is obvious that all points in $M_{s y m}^{(n)}$ are solutions of the Ginzburg-Landau equations, Eq. (1.3).

In order to obtain Eq. (1.1) we add to $\mathcal{E}_{G L}$ an interaction term of the form

$$
\mathcal{E}_{\text {int }}(u)=\int d^{2} x W(x)|\psi(x)|^{2}
$$

where $u \in X^{(n)}$. Define a modified energy functional 


$$
\mathcal{E}_{\epsilon}(u)=\mathcal{E}_{G L}(u)+\epsilon \mathcal{E}_{\text {int }}(u) .
$$

With this definition Eq. (1.1) can be written

$$
\partial_{t} u=-\mathcal{E}_{\epsilon}^{\prime}(u)
$$

where $\mathcal{E}_{\epsilon}^{\prime}$ is the Fréchet derivative of $\mathcal{E}_{\epsilon}$. We observe that the interaction term in Eq. (1.10) does not break the gauge symmetry. However, $\mathcal{E}_{\text {int }}$ does break the translation symmetry and if we translate $\psi$ the interaction energy depends on the translation parameter $\mathbf{z}$. In particular, applying translations to the $n$-vortex solution $v^{(n)}$ we define the interaction energy

$$
W_{i n t}^{(n)}(\mathbf{z}) \equiv \epsilon \int d^{2} x W(\mathbf{x})\left|\psi^{(n)}(\mathbf{x}-\mathbf{z})\right|^{2} .
$$

\subsection{Summary of results}

The main result in this work states that the force derived from the interaction energy $W_{\text {int }}^{(n)}(\mathbf{z})$ is governing the effective dynamics of the magnetic vortex. More specifically, given an initial data $u_{0}=\left(\psi_{0}, A_{0}\right)$ close to a point $v_{\mathbf{z}_{0} \gamma_{0}} \in M_{s y m}^{(n)}$, we show that there exists a curve in $M_{s y m}^{(n)}$, parametrized by the time $t$, such that for all $t \geq 0$ the solution $u(t)$ satisfies

$$
u(t)-v_{\mathbf{z}(t) \gamma(t)}=O(\epsilon)
$$

where the equation for the effective dynamics of the vortex center is given by

$$
a_{n} \dot{\mathbf{z}}+\nabla_{\mathbf{z}} W_{\text {int }}^{(n)}(\mathbf{z})=O\left(\epsilon^{2}\right)
$$

and

$$
a_{n}=\frac{1}{2}\left\|\nabla_{A^{(n)}} \psi^{(n)}\right\|_{2}^{2}+\left\|\nabla \times A^{(n)}\right\|_{2}^{2} .
$$

The rest of the paper is organized as follows: In Section 2 we state the main result proved in this paper in the form of Theorem A. This theorem provides the exact form of the effective dynamics of a magnetic vortex in the presence of a localized potential. In Section 3 we give the proof of Theorem A. Starting in Subsection 3.1 and Subsection 3.2 with the definition and discussion of the notions of manifold of approximate solutions and local Sobolev spaces, we continue in Subsection 3.3 with the four main steps of the proof of Theorem A. A proof of Proposition D, which contains many technical details, is provided in Section 4. Proofs of several technical lemmas are given in Section 5. The proofs of the two important lemmas, Lemma C and Lemma D, are found in Subsection 5.1. Subsection 5.2 contains the proofs of some auxiliary technical lemmas. Finally, Appendix A contains explicit expressions for the Taylor expansions of the energy functional and its Fréchet derivative (r.h.s. of the equation of motion).

\section{Results}




\subsection{Effective dynamics of vortices - main theorem}

Our main result in this work is the following theorem concerning the effective dynamics of the gradient flow Ginzburg-Landau equations with a local potential:

Theorem A (effective dynamics): Let $v^{(n)}=\left(\psi^{(n)}, A^{(n)}\right)$ be an $n$-vortex solution of the static GL equations, Eq. (1.3), with $n$ an arbitrary integer if $0<\lambda<1$ and $n= \pm 1$

if $\lambda>1$. Let $M_{\text {sym }}^{(n)}$ be the symmetry manifold for $v^{(n)}$. Let $u_{0}=\left(\psi_{0}, A_{0}\right)$ be the initial data for a solution $u(t)=(\psi(t), A(t))$ of Eq. (1.1). There exist constants $\epsilon_{0}, c, c_{0}>0$, with $c, c_{0}$ depending only on $\epsilon_{0}$, such that for $0<\epsilon<\epsilon_{0}$ and initial data $u(0)=u_{0}$ satisfying the condition that there is some $v_{\mathbf{z}_{0} \gamma_{0}} \in M_{\text {sym }}^{(n)}$ with

$$
\left\|u_{0}-v_{\mathbf{z}_{0} \gamma_{0}}\right\|_{X^{(n)}} \leq c_{0} \epsilon
$$

the solution $u(t)$ of Eq. (1.1) is of the form

$$
u(t)=v_{\mathbf{z}(t) \gamma(t)}+\zeta(t)
$$

with $v_{\mathbf{z}(t) \gamma(t)} \in M_{\text {sym }}^{(n)}$ and the functions $z(t), \gamma(t)$ satisfying the differential equations

$$
\begin{aligned}
a_{n} \frac{d \mathbf{z}}{d t} & =-\nabla_{\mathbf{z}} W_{i n t}^{(n)}(\mathbf{z})+O\left(\epsilon^{2}\right) \\
\frac{d \gamma}{d t} & =\sum_{i} \dot{z}_{i} A_{i}^{(n)}(\cdot-\mathbf{z})+O_{H^{1-s}}\left(\epsilon^{2}\right), \quad s>0
\end{aligned}
$$

and the function $\zeta(t)$ is in $X^{(n)}$ and is bounded by

$$
\|\zeta(t)\|_{X^{(n)}} \leq c \epsilon
$$

Eq. (2.1) will be called the effective dynamics equations. We see that, for functions $\mathbf{z}(t)$ and $\gamma(t)$ satisfying the effective dynamics equations, $v_{\mathbf{z}(t) \gamma(t)} \in M_{s y m}^{(n)}$ follows the path of the actual solution $u(t)$ up to an error of order $\epsilon$.

\section{Proofs}

Before commencing with the proof of Theorem A we make some comments concerning notation. Throughout the discussion below the symbol $H^{s}$ always means the Sobolev space $H^{s}\left(R^{2} ; C \times R^{2}\right)$. Let $\zeta=(\xi, F) \in L^{2}\left(R^{2} ; C \times R^{2}\right)$ and $\eta=(\lambda, G) \in L^{2}\left(R^{2} ; C \times R^{2}\right)$. The $L^{2}$ inner product of $\zeta$ and $\eta$ is denoted by $\langle\zeta, \eta\rangle$ and is given by

$$
\langle\zeta, \eta\rangle \equiv \int_{R^{2}}(\operatorname{Re}(\bar{\xi} \lambda)+F \cdot G)
$$

In order to make the notation less cubersome we shall denote below the parameters $\{\mathbf{z}, \gamma\}$ by $\sigma$. For example, according to this convention $v_{\sigma}$ stands for $v_{\mathbf{z} \gamma}$. We restore the full notation whenever this is necessary or if it adds to the clarity of argument. Otherwise, the 
shorter notation is kept throughout the course of the proof. In several steps that do not require reference to the parametrization of $M_{a s}$ we omit it altogether.

\subsection{The manifold of approximate solutions}

Let

$$
\Sigma=\left\{(\mathbf{z}, \gamma) \mid \mathbf{z} \in R^{2}, \gamma-\sum_{i} z_{i} A_{i}^{(n)}(\cdot-\mathbf{z}) \in H^{2}\left(R^{2} ; R\right)\right\}
$$

Denote the elements of the translation group by $G_{\mathbf{z}}\left(\mathbf{z} \in R^{2}\right)$ and the elements of the gauge group by $G_{\gamma}\left(\gamma \in H^{2}\left(R^{2} ; C\right)\right)$. Applying the translation and gauge transformations to a vortex solution $v^{(n)}$ of Eq. (1.3) we obtain a manifold $M_{a s}$ of solutions of this equation

$$
M_{a s}=\left\{G_{\gamma} G_{\mathbf{z}} v^{(n)} \mid(\mathbf{z}, \gamma) \in \Sigma\right\}=\left\{v_{\mathbf{z}, \gamma} \mid(\mathbf{z}, \gamma) \in \Sigma\right\}=M_{s y m}^{(n)} \subset X^{(n)}
$$

The manifold $M_{a s}$ is, therefore, parametrized by $\mathbf{z}$ and $\gamma$. A point in $M_{a s}$ corresponding to a given value $\{\mathbf{z}, \gamma\}$ of the parameters (for $\gamma$ this means a definite gauge function) is denoted by $v_{\mathbf{z} \gamma}$.

The manifold $M_{a s}$ is called manifold of approximate solutions. We argue for the introduction of $M_{a s}$ and the terminology used as follows: Inserting $v_{\mathbf{z} \gamma} \in M_{a s}$ as a trial solution into Eq. (1.12) we obtain in the r.h.s

$$
\mathcal{E}_{\epsilon}^{\prime}\left(v_{\mathbf{z} \gamma}\right)=\mathcal{E}_{G L}^{\prime}\left(v_{\mathbf{z} \gamma}\right)+\epsilon \mathcal{E}_{i n t}^{\prime}\left(v_{\mathbf{z} \gamma}\right)=\epsilon \mathcal{E}_{i n t}^{\prime}\left(v_{\mathbf{z} \gamma}\right)
$$

hence the r.h.s of Eq. (1.12) (or, equivalently, of Eq. (1.1)) is of order $\epsilon$ and $v_{\mathbf{z} \gamma}$ is seen to be an approximate static solution of Eq. (1.1) for $\epsilon$ small.

Denote by $T_{\mathbf{z} \gamma} \Sigma$ the tangent space to $\Sigma$ at the point $(\mathbf{z}, \gamma)$ and by $T_{v_{\mathbf{z} \gamma}} M_{a s}$ the tangent space to $M_{a s}$ at the point $v_{\mathbf{z} \gamma}$. A basis for $T_{\mathbf{z} \gamma} \Sigma$ is given by

$$
\left\{\partial_{z_{i}}+\left\langle\mathbf{z} \cdot \partial_{i} A, \partial_{\tilde{\gamma}}\right\rangle, \partial_{\tilde{\gamma}}\right\}
$$

where $\tilde{\gamma}$ is given by Eq. (1.8) and for a function $g$ we set $\left\langle g, \partial_{\tilde{\gamma}}\right\rangle=\int d^{2} x g(x) \partial_{\tilde{\gamma}(x)}$. Using the natural parametrization map $\beta: \Sigma \rightarrow M_{a s}$ defined by $\beta(\mathbf{z}, \gamma)=v_{\mathbf{z} \gamma}$ we can push forward the basis given in Eq. (3.4) in order to obtain a basis for $T_{v_{\mathbf{z} \gamma}} M_{a s}$. This later basis, is obtained via the mapping of the basis in Eq. (3.4) by the Fréchet derivative $D_{\mathbf{z} \gamma}(\beta(\mathbf{z}, \gamma))=D_{\mathbf{z} \gamma} v_{\mathbf{z} \gamma}$. Denoting $\partial_{z_{i}}^{A}=\partial_{z_{i}}+\left\langle A_{i}, \partial_{\gamma}\right\rangle$, we get in $T_{v_{\mathbf{z} \gamma}} M_{a s}$ the basis vectors

$$
T_{i}^{\mathbf{z} \gamma}=\partial_{z_{i}}^{A} v_{\mathbf{z} \gamma}, i=1,2 \quad G_{\delta(x)}^{\mathbf{z} \gamma}=\partial_{\gamma(x)} v_{\mathbf{z} \gamma}
$$

where the (covariant) translation vectors $T_{i}^{\mathbf{z} \gamma}$ are given by

$$
T_{i}^{\mathbf{z} \gamma}=\left(\left(\nabla_{A_{\mathbf{z} \gamma}} \psi_{\mathbf{z} \gamma}\right)_{i},\left(\nabla \times A_{\mathbf{z} \gamma}\right) \hat{e}_{i}\right) .
$$

Here $\hat{e}_{1}=(0,1)$ and $\hat{e}_{2}=(-1,0)$. For a definite gauge function $\chi$ the gauge basis vectors $G_{\delta(x)}^{\mathbf{z} \gamma}$ in Eq. (3.5) are defined by the relation

$$
\int d^{2} x G_{\delta(x)}^{\mathbf{z} \gamma} \chi(x)=G_{\chi}^{\mathbf{z} \gamma}
$$


where

$$
G_{\chi}^{\mathbf{z} \gamma}=\left(i \chi \psi_{\mathbf{z} \gamma}, \nabla \chi\right)
$$

The vectors in Eq. (3.5) form an orthogonal basis for $T_{v_{\mathbf{z} \gamma}} M_{a s}$. We have $\left\langle G_{\delta(x)}^{\mathbf{z} \gamma}, T_{i}^{\mathbf{z} \gamma}\right\rangle=0$ and $\left\langle T_{i}^{\mathbf{z} \gamma}, T_{j}^{\mathbf{z} \gamma}\right\rangle=\delta_{i j} a_{n}$ with

$$
a_{n}=\left\|T_{1}^{\mathbf{z} \gamma}\right\|_{2}^{2}=\left\|T_{2}^{\mathbf{z} \gamma}\right\|_{2}^{2}=\frac{1}{2}\left\|\nabla_{A^{(n)}} \psi^{(n)}\right\|_{2}^{2}+\left\|\nabla \times A^{(n)}\right\|_{2}^{2}
$$

and, in addition

$$
\left\langle G_{\delta(x)}^{\mathbf{z} \gamma}, G_{\delta(y)}^{\mathbf{z} \gamma}\right\rangle=K_{\mathbf{z} \gamma}(x, y)
$$

where $K_{\mathbf{z} \gamma}(x, y)$ is the integral kernel for the operator

$$
K_{\mathbf{z} \gamma}=-\Delta+\left|\psi_{\mathbf{z} \gamma}\right|^{2}
$$

We note that $K_{\mathbf{z} \gamma}$ is self-adjoint and that $K_{\mathbf{z} \gamma}>0$.

We will see below that one of the main ingredients that enter into the proof of Theorem $\mathrm{A}$ is the projection of Eq. (1.1), for each time $t$, on the tangent space $T_{v_{\mathbf{z} \gamma}} M_{a s}$ (where $\mathbf{z}$ and $\gamma$ depend on $t$ in a sutible way). Denote $P_{v}: T_{v} X^{(n)} \rightarrow T_{v} M_{a s}$ the projection on the tangent space to $M_{a s}$ at the point $v_{\mathbf{z} \gamma}$. We are able to use the orthogonal basis of Eq. (3.5) to obtain an explicit expression for $P_{v_{\mathbf{z} \gamma}}$. For any $\zeta \in T_{v_{\mathbf{z} \gamma}} X^{(n)}$ we have

$$
P_{v_{\mathbf{z} \gamma}} \zeta=\sum_{i=1,2} a_{n}^{-1} T_{i}^{\mathbf{z} \gamma}\left\langle T_{i}^{\mathbf{z} \gamma}, \zeta\right\rangle+\int d^{2} x \int d^{2} y G_{\delta(x)}^{\mathbf{z} \gamma} K_{\mathbf{z} \gamma}^{-1}(x, y)\left\langle G_{\delta(y)}^{\mathbf{z} \gamma}, \zeta\right\rangle
$$

In particular, if $v(t)=v_{\mathbf{z}(t) \gamma(t)}$ is a path in $M_{a s}$, depending on the time $t$, then we have

$$
\dot{v}_{\mathbf{z} \gamma}=P_{v} \dot{v}_{\mathbf{z} \gamma}=\sum_{i}\left(-\dot{z}_{i}\right) T_{i}^{\mathbf{z} \gamma}+G_{\dot{\tilde{\gamma}}}^{\mathbf{z} \gamma}
$$

with

$$
\dot{\tilde{\gamma}}=\dot{\gamma}-\sum_{i} \dot{z}_{i} A_{i}^{(n)}(\cdot-\mathbf{z})
$$

In Eq. (3.13) and Eq. (3.14) we use the notation $\dot{v}_{\mathbf{z} \gamma}=\partial_{t} v_{\mathbf{z} \gamma}, \dot{\gamma}=\partial_{t} \gamma, \dot{\tilde{\gamma}}=\partial_{t} \tilde{\gamma}$ and $\dot{\mathbf{z}}=\partial_{t} \mathbf{z}$.

\subsection{Local Sobolev spaces on the manifold of approximate solutions}

In this subsection we define the notion of local Sobolev spaces on the manifold of approximate solutions $M_{a s}$. The introduction of this concept helps to facilitate many of the estimates in this work and is based on the observation that certain estimates are not uniform on $M_{a s}$ but depend on the point $v \in M_{a s}$. More specifically, at the point $v_{\mathbf{z} \gamma}$ the constants appearing in the estimates depend on the gauge function $\gamma$. 
As an example for the motivation for the definition of local Sobolev spaces on $M_{a s}$ we give an estimate which is used below in the proof of Lemma B. Consider vectors $(\xi, F) \in$ $T_{v_{\mathbf{z} \gamma}} X^{(n)}$ with the component $\xi$ transforming covariantly under gauge transformations and $F$ gauge invariant. Let $\zeta=(\xi, F)$ be such a vector and let $G_{\gamma} \zeta$ be the action of (an appropriate unitary representation of) an element $G_{\gamma}$ of the gauge group on $\zeta$. We have $G_{\gamma} \zeta=\zeta^{\prime}$ where $\zeta^{\prime}=\left(\xi^{\prime}, F^{\prime}\right)=\left(e^{i \gamma} \xi, F\right)$. Construct a vector $\zeta_{v}=\left(0, \operatorname{Im}\left(\bar{\xi} \nabla_{A} \xi\right)\right)$ where $\xi$ is the first component of $\zeta$. Then $\zeta_{v} \in T_{v_{\mathbf{z} \gamma}} X^{(n)}$ has the desired transformation properties under the action of the gauge group. Suppose that we want to estimate the $H^{-s}(s>0)$ norm of $\zeta_{v}$. we have

$$
\left\|\zeta_{v}\right\|_{H^{-s}}=\left\|\bar{\xi} \nabla_{A} \xi\right\|_{H^{-s}}=\left\|\bar{\xi} \nabla \xi-i A|\xi|^{2}\right\|_{H^{-s}} \leq\|\bar{\xi} \nabla \xi\|_{H^{-s}}+\left\|A|\xi|^{2}\right\|_{H^{-s}}
$$

For the first term on the r.h.s. of Eq. (3.15) we have the estimate

$$
\begin{aligned}
\|\bar{\xi} \nabla \xi\|_{H^{-s}} & =\sup _{\|\eta\|_{H^{s}} \leq 1}|\langle\eta, \bar{\xi} \nabla \xi\rangle|=\sup _{\|\eta\|_{H^{s}} \leq 1}|\langle\eta \xi, \nabla \xi\rangle| \leq \sup _{\|\eta\|_{H^{s}} \leq 1}\|\eta \xi\|_{2}\|\nabla \xi\|_{2} \\
& \leq \sup _{\|\eta\|_{H^{s}} \leq 1}\|\eta\|_{p}\|\xi\|_{q}\|\xi\|_{H^{1}} \leq\|\xi\|_{H^{1}}^{2}
\end{aligned}
$$

where $1 / 2=1 / p+1 / q$ and $q$ is large enough so that $H^{s} \subset L^{p}$. In order to estimate the second term on the r.h.s. of Eq. (3.15) we recall that at $v_{\mathbf{z} \gamma}$ we have $A_{\mathbf{z} \gamma}=A^{(n)}(\cdot-\mathbf{z})+\nabla \gamma$ and so

$$
\left\|A|\xi|^{2}\right\|_{H^{-s}}=\left\|\left(A^{(n)}(\cdot-\mathbf{z})+\nabla \gamma\right)|\xi|^{2}\right\|_{H^{-s}} \leq\left\|A^{(n)}\right\|_{\infty}\|\xi\|_{2}^{2}+\left\|\nabla \gamma|\xi|^{2}\right\|_{H^{-s}}
$$

For the second term on the r.h.s. of Eq. (3.17) we have the estimate

$$
\begin{aligned}
\left\|\nabla \gamma|\xi|^{2}\right\|_{H^{-s}} & =\sup _{\|\eta\|_{H^{s}} \leq 1}\left|\left\langle\eta, \nabla \gamma|\xi|^{2}\right\rangle\right|=\sup _{\|\eta\|_{H^{s}} \leq 1}\left|\left\langle\eta|\xi|^{2}, \nabla \gamma\right\rangle\right| \leq \sup _{\|\eta\|_{H^{s}} \leq 1}\left\|\left.\left.\eta\right|^{2}\right|^{2}\right\|_{2}\|\nabla \gamma\|_{2} \\
& \leq \sup _{\|\eta\|_{H^{s}} \leq 1}\|\eta\|_{p}\left\|\xi^{2}\right\|_{q}\|\gamma\|_{H^{1}} \leq\|\xi\|_{2 q}^{2}\|\gamma\|_{H^{1}} \leq\|\xi\|_{H^{1}}^{2}\|\gamma\|_{H^{1}}
\end{aligned}
$$

where again $1 / 2=1 / q+1 / p$ and $q$ is large enough so that $H^{s} \subset L^{p}$. Using Eq. (3.16)-(3.18) we obtain

$$
\left\|\zeta_{v}\right\|_{H^{-s}} \leq C_{\gamma}\left\|\zeta_{v}\right\|_{H^{1}}^{2}
$$

where

$$
C_{\gamma}=C\left(1+\|\gamma\|_{H^{1}}\right)
$$

we see that this estimate is not uniform on $M_{a s}$ but depends on the gauge function $\gamma$.

In order to simplify estimates of the type considered here we define the notion of local Sobolev spaces on $M_{a s}$. With a point $v_{\mathbf{z} \gamma} \in M_{a s}$, and for any real $s$, we associate a local 
Sobolev space $H_{v_{\mathbf{z} \gamma}}^{s}$ such that, given $\zeta=(\xi, F) \in T_{v_{\mathbf{z} \gamma}} X^{(n)}$ with the gauge transformation properties described above, its $H_{v_{\mathbf{z} \gamma}}^{s}$ norm is defined to be

$$
\|\zeta\|_{H_{v_{\mathbf{z} \gamma}}} \equiv\left\|G_{-\gamma} \zeta\right\|_{H^{s}}
$$

The definition of $H_{v_{\mathbf{z} \gamma}}^{s}$ preserves all of the properties of Sobolev spaces. In particular, the Sobolev embedding theorems are all valid for the local Sobolev spaces. Note also that $L_{v_{\mathbf{z} \gamma}}^{p}=L^{p}$ for all $p$.

Suppose we want to obtain an estimate for the vector $\zeta_{v}=\left(0, \operatorname{Im}\left(\bar{\xi} \nabla_{A} \xi\right)\right)$ in the local $H_{v_{\mathbf{z} \gamma}}^{-s}(s>0)$ norm. We have

$$
\left\|\zeta_{v}\right\|_{H_{v_{\mathbf{z} \gamma}}^{-s}}=\left\|\bar{\xi} \nabla_{A} \xi\right\|_{H^{-s}}=\left\|\left(\overline{e^{-i \gamma} \xi}\right)\left(e^{-i \gamma} \nabla_{A} \xi\right)\right\|_{H^{-s}}=\left\|\overline{\xi^{\prime}} \nabla_{A^{(n)}} \xi^{\prime}\right\|_{H^{-s}}
$$

where $\xi^{\prime}=e^{-i \gamma} \xi$. Repeating the sequence of estimates in Eq. (3.16)-(3.18) for the r.h.s. of Eq. (3.22) we find that

$$
\left\|\overline{\xi^{\prime}} \nabla_{A^{(n)}} \xi^{\prime}\right\|_{H^{-s}} \leq C\left\|\xi^{\prime}\right\|_{H^{1}}^{2}=C\left\|e^{-i \gamma} \xi\right\|_{H^{1}}^{2}=C\|\xi\|_{H_{v_{\mathbf{z} \gamma}}^{1}}^{2}
$$

with $C=1+\left\|A^{(n)}\right\|_{\infty}$, a constant independent of $\gamma$. Thus we arrive at the (local) estimate

$$
\left\|\zeta_{v}\right\|_{H_{v_{\mathbf{z} \gamma}}^{-s}} \leq C\left\|\zeta_{v}\right\|_{H_{v_{\mathbf{z} \gamma}}^{1}}^{2}
$$

which has the same form as Eq. (3.19). The emphasis is on the fact that the constant $C$ here does not depend on $\gamma$.

In accord with the discussion here most of the estimates involved in the proof of the main theorem of this work are performed using the local Sobolev spaces $H_{v_{\mathbf{z} \gamma}}^{s}$.

\subsection{Proof of Theorem A}

The strategy for the proof of Theorem A is as follows: Given $\epsilon>0$ small enough we show that there exists a neighborhood of $M_{a s}$ such that, if the initial data $u_{0}$ for Eq. (1.1) is in this neighborhood, then the distance of the solution $u(t)$ from $M_{a s}$ can be controlled for all times $t \geq 0$. For such a solution we define in a sutible way a projection on $M_{a s}$ so as to obtain, for each $t \geq 0$ a unique point $v(t)=v(u(t)) \in M_{a s}$ corresponding to $u(t)$. We think of $u(t)$ as inducing a (uniquely defined) trajectory on $M_{a s}$, traced by $v(t)$. We then obtain effective equations of motion for the point $v(t)$ in terms of the parametrization of $M_{a s}$ by the translation parameters $\mathbf{z}$ and the gauge function $\gamma$, i.e, we obtain equations of motion for these parameters and prove their accuracy to order $\epsilon^{2}$ for all $t \geq 0$. The process is considered as a reduction of the problem of the analysis of the dynamics generated by Eq. (1.1) to a dynamical problem consisting of a small number (in a sense) of degrees of freedom, providing an effective overall behavior of the original system.

As mentioned above, the proof of Theorem A consists of several steps:

Step 1- Decomposition 
the first step in the proof is to establish the validity an appropriate decomposition for any $u \in X^{(n)}$ which is close enough to $M_{a s}$ :

Proposition A (decomposition): Define a neighborhood $U_{\delta} \subset X^{(n)}$ of $M_{a s}$ in $X^{(n)}$ as follows

$$
U_{\delta}=\left\{u \mid u \in X^{(n)} \text { and } \exists(\mathbf{z}, \gamma) \in \Sigma,\left\|u-v_{\mathbf{z} \gamma}\right\|_{X^{(n)}}<\delta\right\} .
$$

Then there exist $\delta>0$ and a $C^{1}$ map $M_{d e c}: U_{\delta} \mapsto \Sigma$ such that, if we denote $v(u)=$ $\beta\left(M_{\text {dec }}(u)\right)$ for $u \in U_{\delta}$ (where $\beta(\mathbf{z}, \gamma)=v_{\mathbf{z} \gamma}$; the parametrization mapping for $\left.M_{a s}\right)$, we have $u-v(u) \in H^{1}\left(R^{2} ; C \times R^{2}\right)$ and

$$
P_{v(u)}(u-v(u))=0
$$

\section{Proof:}

Given the expression in Eq. (3.12) for the projection operator $P_{v_{\mathbf{z} \gamma}}$ we see that condition (3.26) is equivalent to the following conditions

$$
\left\langle T_{i}^{\mathbf{z} \gamma},\left(u-v_{\mathbf{z} \gamma}\right)\right\rangle=0, i=1,2 \quad\left\langle G_{\delta_{(x)}}^{\mathbf{z} \gamma},\left(u-v_{\mathbf{z}, \gamma}\right)\right\rangle=0
$$

Define a function $g: U_{\delta} \times \Sigma \rightarrow R^{2} \times L^{2}\left(R^{2}\right)$ by

$$
g(u ; \mathbf{z}, \gamma)=\left(\left\langle T_{i}^{\mathbf{z} \gamma},\left(u-v_{\mathbf{z} \gamma}\right)\right\rangle,\left\langle G_{\delta_{(x)}}^{\mathbf{z} \gamma},\left(u-v_{\mathbf{z} \gamma}\right)\right\rangle\right)
$$

It is obvious that $g\left(v_{\sigma} ; \sigma\right)=0$. Taking the Fréchet derivative we obtain a map $D_{\sigma} g\left(v_{\sigma} ; \sigma\right)$ : $T_{\sigma} \Sigma \mapsto R^{2} \times L^{2}\left(R^{2}\right)$

$$
D_{\sigma} g\left(v_{\sigma} ; \sigma\right)=-\left(\left\langle T_{i}^{\sigma}, D_{\sigma} v_{\sigma}\right\rangle,\left\langle G_{\delta_{(x)}}^{\sigma}, D_{\sigma} v_{\sigma}\right\rangle\right)
$$

Using the basis of $T_{\sigma} \Sigma$ given in Eq. (3.4) we can express Eq. (3.28) as a transformation on the coordinate vector in that basis. We then obtain a matrix representation $\left[D_{\sigma} g\left(v_{\sigma} ; \sigma\right)\right]_{R}$ of $D_{\sigma} g\left(v_{\sigma} ; \sigma\right)$ with

$$
\left[D_{\sigma} g\left(v_{\sigma} ; \sigma\right)\right]_{R}: R^{2} \times H^{2}\left(R^{2} ; R\right) \mapsto R^{2} \times L^{2}\left(R^{2}\right)
$$

and

$$
\left[D_{\sigma} g\left(v_{\sigma} ; \sigma\right)\right]_{R}=\operatorname{diag}\left\{-a_{n},-a_{n}, K_{\sigma}\right\} .
$$

Since $K_{\sigma}$ is invertible we find that $\left[D_{\sigma} g\left(v_{\sigma} ; \sigma\right)\right]_{R}$ is invertible (hence also $D_{\sigma} g\left(v_{\sigma} ; \sigma\right)$ ). The implicit function theorem then implies that for any $v_{\sigma} \in M_{a s}$ there is a unique $C^{1}$ map $M_{d e c}$ from $B_{X^{(n)}}\left(v_{\sigma} ; \delta\right)$, a ball in $X^{(n)}$ of size $\delta$ centered at $v_{\sigma}$, to $\Sigma$ such that, for $\sigma=M_{d e c}(u)$, Eq. (3.27) and hence Eq. (3.26) are satisfied. We note that $\left\|K_{\sigma}^{-1}\right\|_{L^{2} \rightarrow H^{2}}$ is uniformly bounded on $M_{a s}$ and also that $D_{\sigma} g(u ; \sigma)$ and $D_{\sigma}^{2} g(u ; \sigma)$ contain only covariant derivatives of $T_{i}^{\sigma}$ and $G_{\delta_{(x)}}^{\sigma}$. We therefore find that the coordinate transformation representations

$$
\left[D_{\sigma} g(u ; \sigma)\right]_{R}: X^{(n)} \times\left(R^{2} \times H^{2}\left(R^{2} ; R\right)\right) \mapsto R^{2} \times L^{2}\left(R^{2}\right)
$$


and

$$
\left[D_{\sigma}^{2} g(u ; \sigma)\right]_{R}: X^{(n)} \times\left(R^{2} \times H^{2}\left(R^{2} ; R\right)\right) \times\left(R^{2} \times H^{2}\left(R^{2} ; R\right)\right) \mapsto R^{2} \times L^{2}\left(R^{2}\right)
$$

are uniformly bounded in $\sigma$ for all balls $B_{X^{(n)}}\left(v_{\sigma} ; \delta_{0}\right)$, where $\delta_{0}$ is independent of $\sigma$. Thus, we can choose $\delta$ independent of the point $v_{\sigma}$ and the existence of an appropriate neighborhood $U_{\delta}$ is established.

Consider a solution $u(t)$ of Eq. (1.1) which, for a time interval $t \in\left[0, T_{\delta}\right]$ satisfies $u(t) \in U_{\delta}$. Proposition A then implies that for such a solution it is possible to find, for each $t \in\left[0, T_{\delta}\right]$ a unique point $v(u(t)) \in M_{a s}$ such that the condition in Eq. (3.26) holds. If we denote $\zeta_{v}(t)=u(t)-v(u(t))$ then $\zeta(t) \in X^{(n)}$ and we obtain a unique decomposition

$$
u(t)=v(u(t))+\zeta_{v}(t)
$$

with

$$
P_{v(u(t))} \zeta_{v}(t)=0 .
$$

For the sake of bravity we omit in the sequel from our notation the time $t$ and all indications for the procedure of obtaining the point $v(u(t))$ when given a solution $u(t)$. Thus we write, unless a temporary need arises to restore the full notation, $u(t), v(u(t))$ and $\zeta_{v}(t)$ as $u, v$ and $\zeta_{v}$ respectively. Eq. (3.31) will then be written in short as $u=v+\zeta_{v}$, Eq. (3.32) will be written $P_{v} \zeta_{v}=0$, etc.

\section{Step 2- Effective dynamics equations}

Once the existence of the decomposition, Eq. (3.31)-(3.32), is established, we can project Eq. (1.1) on the tangent space to $M_{a s}$ in order to obtain the effective equations of motion. Applying the projection $P_{v}$ to Eq. (1.12) we get

$$
P_{v} \dot{u}=-P_{v} \mathcal{E}_{\epsilon}^{\prime}(u)
$$

Making use of the decomposition in Eq. (3.31) we obtain

$$
P_{v} \dot{v}=-P_{v} \mathcal{E}_{\epsilon}^{\prime}(u)-P_{v} \dot{\zeta}_{v}
$$

We expand in a Taylor series the Fréchet derivative of the energy functional

$$
\mathcal{E}_{\epsilon}^{\prime}(u)=\mathcal{E}_{\epsilon}^{\prime}(v)+L_{\epsilon ; v} \zeta_{v}+N_{v}\left(\zeta_{v}\right)
$$

where $L_{\epsilon ; v} \equiv \mathcal{E}_{\epsilon}^{\prime \prime}(v)$ and $N_{v}\left(\zeta_{v}\right)$ is the non-linear term defined by $N_{v}\left(\zeta_{v}\right)=\mathcal{E}_{\epsilon}^{\prime}(u)-\mathcal{E}_{\epsilon}^{\prime}(v)-$ $L_{\epsilon ; v} \zeta_{v}$. Inserting Eq. (3.35) into Eq. (3.34) we get

$$
P_{v} \dot{v}=-P_{v}\left(\mathcal{E}_{\epsilon}^{\prime}(v)+L_{\epsilon ; v} \zeta_{v}+N_{v}\left(\zeta_{v}\right)\right)-P_{v} \dot{\zeta}_{v}
$$

Note further that $P_{v} \dot{v}=\dot{v}$ (since $\dot{v} \in T_{v} M_{a s}$ ) and hence 


$$
\dot{v}+P_{v} \mathcal{E}_{\epsilon}^{\prime}(v)=-P_{v} L_{\epsilon ; v} \zeta_{v}-P_{v} N_{v}\left(\zeta_{v}\right)-P_{v} \dot{\zeta}_{v}
$$

The last term on the r.h.s. of Eq. (3.37) can be written in a more convenient form by using Eq. (3.32). We have

$$
0=\partial_{t}\left(P_{v} \zeta_{v}\right)=\dot{P}_{v} \zeta_{v}+P_{v} \dot{\zeta}_{v}
$$

and so

$$
P_{v} \dot{\zeta}_{v}=-\dot{P}_{v} \zeta_{v}
$$

Inserting Eq. (3.38) into Eq. (3.37) we obtain

$$
\dot{v}+P_{v} \mathcal{E}_{\epsilon}^{\prime}(v)=-P_{v} L_{\epsilon ; v} \zeta_{v}-P_{v} N_{v}\left(\zeta_{v}\right)+\dot{P}_{v} \zeta_{v} .
$$

Eq. (3.39) is the starting point for the derivation of the effective dynamics on the manifold $M_{a s}$ induced by the gradient flow equations.

We begin by estimating Eq. (3.39)

Proposition B: Suppose that a solution $u$ of Eq. (1.1) satisfy the condition that $u(t) \in U_{\delta}$ for $t \in\left[0, T_{\delta}\right]$, where $U_{\delta} \subset X^{(n)}$ is the neighborhood of $M_{a s}$ given by Proposition A. For such $u$ the decomposition $u=v+\zeta_{v}$ is valid and we have the following estimate for $t \in\left[0, T_{\delta}\right]$

$$
\left\|\dot{v}_{\sigma}+P_{v_{\sigma}} \mathcal{E}_{\epsilon}^{\prime}\left(v_{\sigma}\right)\right\|_{H_{v_{\sigma}}^{-s}} \leq C\left[\epsilon\left\|\zeta_{v_{\sigma}}\right\|_{H_{v_{\sigma}}^{1}}+\left\|\zeta_{v_{\sigma}}\right\|_{H_{v_{\sigma}}^{1}}^{2}+\left\|\zeta_{v_{\sigma}}\right\|_{H_{v_{\sigma}}^{1}}^{3}+\left(|\dot{\mathbf{z}}|+\|\dot{\tilde{\gamma}}\|_{2}\right)\left\|\zeta_{v_{\sigma}}\right\|_{H_{v_{\sigma}}^{1}}\right]
$$

for some $C>0$.

\section{Proof:}

A first estimate of Eq. (3.39) gives

$$
\left\|\dot{v}_{\sigma}+P_{v_{\sigma}} \mathcal{E}_{\epsilon}^{\prime}\left(v_{\sigma}\right)\right\|_{H_{v_{\sigma}}^{-s}} \leq\left\|P_{v_{\sigma}} L_{\epsilon ; v_{\sigma}} \zeta_{v_{\sigma}}\right\|_{H_{v_{\sigma}}^{-s}}+\left\|P_{v_{\sigma}} N_{v_{\sigma}}\left(\zeta_{v_{\sigma}}\right)\right\|_{H_{v_{\sigma}}^{-s}}+\left\|\dot{P}_{v_{\sigma}} \zeta_{v_{\sigma}}\right\|_{H_{v_{\sigma}}^{-s}}
$$

The proposition is a result of Lemma A, Lemma B and Lemma C below, which provide estimates on the terms on the r.h.s. of Eq. (3.41):

Lemma A (approximate zero modes property): There exists a constant $C>0$ such that, for all $v \in M_{\text {as }}$ and any vector $\zeta \in T_{v} X^{(n)}$, we have

$$
\left\|P_{v} L_{v ; \epsilon} \zeta\right\|_{2} \leq \epsilon C\|\zeta\|_{2}
$$

where $\epsilon$ is the potential strength parameter appearing in Eq. (1.1).

Lemma A is proved in Subsection 5.2. This lemma provides a bound on the first term on the r.h.s. of Eq. (3.41) (since $L^{2}=L_{v_{\mathbf{z} \gamma}}^{2}$ ). A straightforward technical calculation 
provides an estimate for the middle term on the r.h.s of Eq. (3.41). This is also done in Subsection 5.2, where we obtain the following result:

Lemma B: For $\zeta \in T_{v} X^{(n)}$ and $s>0$ we have

$$
\left\|P_{v_{\sigma}} N_{v_{\sigma}}(\zeta)\right\|_{H_{v_{\sigma}}^{-s}} \leq C\left(\|\zeta\|_{H_{v_{\sigma}}^{1}}^{2}+\|\zeta\|_{H_{v_{\sigma}}^{1}}^{3}\right)
$$

An estimate of the last term on the r.h.s. of Eq. (3.42) is given in Lemma C, proved in Subsection 5.1:

Lemma C: For $\zeta_{v_{\sigma}} \in T_{v_{\sigma}} X^{(n)}$ satisfying $P_{v_{\sigma}} \zeta_{v_{\sigma}}=0$ we have

$$
\left\|\dot{P}_{v_{\sigma}} \zeta_{v_{\sigma}}\right\|_{2} \leq C\left(|\dot{\mathbf{z}}|+\|\dot{\tilde{\gamma}}\|_{2}\right)\left\|\zeta_{v_{\sigma}}\right\|_{H_{v_{\sigma}}^{1}}
$$

where $\dot{\tilde{\gamma}}$ is given in Eq. (3.14).

insertion of the inequalities, Eq. (3.42)-(3.44) into Eq. (3.41) completes the proof of proposition B.

Step 3- A bound on the error term

Given the decomposition $u=v+\zeta_{v}$, for a solution $u$ satisfying the conditions of Proposition A, we show that the remainder term $\zeta_{v}$ is of order $\epsilon$ for all times $t>0$, where $\epsilon$ is the potential strength parameter (this result also justifies the assumption that the decomposition implied by Proposition A exists for all times if the initial data $u(0)$ satisfies certain requirments which are specified below). The following proposition is the main step in the proof of Theorem A:

Proposition C: Let $u(t)$ be a solution of Eq. (1.1) with initial data $u(0)=u_{0}=$ $\left(\psi_{0}, A_{0}\right)$. There exist constants $\epsilon_{0}, c_{0}, c>0$, with $c_{0}$ and $c$ depending only on $\epsilon_{0}$, such that, for any $0<\epsilon<\epsilon_{0}$, if $u_{0}$ is chosen in such a way that there exists a point $v_{\mathbf{z}_{0} \gamma_{0}} \in M_{a s}$ with $\left\|u_{0}-v_{\mathbf{z}_{0} \gamma_{0}}\right\|_{X^{(n)}} \leq c_{0} \epsilon$, then Proposition $A$ holds for $u(t)$ for all $t \geq 0$ and the decomposition $u(t)=v_{\sigma}(t)+\zeta_{v_{\sigma}}(t)$ satisfies

$$
\left\|\zeta_{v_{\sigma}}(t)\right\|_{X^{(n)}} \leq c \epsilon, \quad t \geq 0
$$

and

$$
\left\|\dot{v}_{\sigma}+P_{v_{\sigma}} \mathcal{E}_{\epsilon}^{\prime}\left(v_{\sigma}\right)\right\|_{H_{v_{\sigma}}^{-s}} \leq c \epsilon^{2}, \quad t \geq 0
$$

Proof: The central result underlying the proof of Theorem A is the linear stability Theorem for the GL equations proved by S. Gustafson and I.M. Sigal [GS1]. This linear 
stability property corresponds to the coercivity of the Hessian. We start the proof of proposition $\mathrm{C}$ by stating this theorem in a form convenient for our purposes.

Suppose that the manifold $M_{a s}$ corresponds to a vortex with index $n$, i.e., $M_{a s}=$ $M_{\text {sym }}^{(n)}$. An $n$-vortex is called stable if there exists some constant $\nu>0$ such that

$$
\left.L_{0 ; v}\right|_{\left(T_{v} M_{a s}\right)^{\perp} \geq \nu .}
$$

An $n$-vortex is called unstable if $L_{0 ; v}$ has a negative eigenvalue. We have

\section{Linear Stability Theorem (S. Gustafson and I.M. Sigal):}

(i) For all $\lambda>0$ the vortex with $n= \pm 1$ is stable.

(ii) For $\lambda<1$ a vortex with $|n| \geq 2$ is stable.

(ii) For $\lambda>1$ a vortex with $|n| \geq 2$ is unstable.

In our work here we consider only stable vortices. Then $\left.L_{0 ; v}\right|_{\left(T_{v} M_{a s}\right)^{\perp}}$ is a positive operator. In this case, for any vector $\zeta_{v} \in T_{v} X^{(n)}$ such that $P_{v} \zeta_{v}=0$, the linear stability theorem implies that

$$
\left\langle\zeta_{v}, L_{0 ; v} \zeta_{v}\right\rangle \geq \nu\left\|\zeta_{v}\right\|_{H^{1}}^{2}
$$

for some constant $\nu>0$.

Introducing the potential $W$ along with the potential strength parameter $\epsilon$ we have the following corollary to the linear stability theorem:

Corollary A: For a vector $\zeta_{v} \in T_{v} X^{(n)}$ with $P_{v} \zeta_{v}=0$ and for $\epsilon>0$ we have

$$
\left\langle\zeta_{v}, L_{\epsilon ; v} \zeta_{v}\right\rangle \geq \nu\left\|\zeta_{v}\right\|_{H^{1}}^{2}
$$

Proof: We calculate

$$
\left\langle\zeta_{v}, L_{\epsilon ; v} \zeta_{v}\right\rangle=\left\langle\zeta_{v},\left(L_{0 ; v}+\epsilon W\right) \zeta_{v}\right\rangle=\left\langle\zeta_{v}, L_{0 ; v} \zeta_{v}\right\rangle+\epsilon\left\langle\zeta_{v}, W \zeta_{v}\right\rangle \geq\left\langle\zeta_{v}, L_{0 ; v} \zeta_{v}\right\rangle \geq \nu\left\|\zeta_{v}\right\|_{H^{1}}^{2}
$$

since the term containing the potential $W$ is positive.

The following result, which we use below, is also an immediate corollary of the linear stability theorem have

Corollary B: For a vector $\zeta_{v_{\sigma}} \in T_{v_{\sigma}} X^{(n)}$ satisfying $P_{v_{\sigma}} \zeta_{v_{\sigma}}=0$ and for $\epsilon>0$ we

$$
\left\langle\zeta_{v_{\sigma}}, L_{\epsilon ; v_{\sigma}} \zeta_{v_{\sigma}}\right\rangle \geq \nu\left\|\zeta_{v_{\sigma}}\right\|_{H_{v_{\sigma}}^{1}}^{2}
$$


Proof: It is easy to check that the application of the gauge transformation $G_{-\gamma}$ to the projection $P_{v_{\mathbf{z} \gamma}}$ yields $G_{-\gamma} P_{v_{\mathbf{z} \gamma}} G_{-\gamma}^{*}=G_{-\gamma} P_{v_{\mathbf{z} \gamma}} G_{\gamma}=P_{v_{\mathbf{z} 0}}$. This implies that

$$
0=G_{-\gamma} P_{v_{\sigma}} \zeta_{v_{\sigma}}=G_{-\gamma} P_{v_{\sigma}} G_{-\gamma}^{*} G_{-\gamma} \zeta_{v_{\sigma}}=P_{v_{\mathbf{z} 0}} \zeta_{v_{\mathbf{z} 0}}
$$

where $\zeta_{v_{\mathbf{z} 0}}=G_{-\gamma} \zeta_{v_{\sigma}}$. In addition we have $G_{-\gamma} L_{\epsilon ; v_{\sigma}}=L_{\epsilon ; v_{\mathbf{z} 0}} G_{-\gamma}$. Therefore, we obtain

$$
\begin{aligned}
\left\langle\zeta_{v_{\sigma}}, L_{\epsilon ; v_{\sigma}} \zeta_{v_{\sigma}}\right\rangle & =\left\langle G_{-\gamma} \zeta_{v_{\sigma}}, G_{-\gamma} L_{\epsilon ; v_{\sigma}} \zeta_{v_{\sigma}}\right\rangle \\
& =\left\langle\zeta_{v_{\mathbf{z} 0}}, L_{\epsilon ; v_{\mathbf{z} 0}} \zeta_{v_{\mathbf{z} 0}}\right\rangle \geq \nu\left\|\zeta_{v_{\mathbf{z} 0}}\right\|_{H^{1}}^{2}=\nu\left\|G_{-\gamma} \zeta_{v_{\sigma}}\right\|_{H^{1}}^{2}=\nu\left\|\zeta_{v_{\sigma}}\right\|_{H_{v_{\sigma}}^{1}}^{2}
\end{aligned}
$$

In the proof of proposition $\mathrm{C}$ we make use of the dominating linear stability properties mentioned above by analyzing the time evolution and providing an upper bound on the quantity $\left\langle\zeta_{v}, L_{\epsilon ; v} \zeta_{v}\right\rangle$. Corollary $\mathrm{A}$ of the linear stability theorem then ensures that this yields a bound on the error term $\zeta_{v}$.

In Section 4 we prove the following proposition:

Proposition D: Let $u$ be a solution of Eq. (1.1) satisfying the assumption of Proposition $B$ above and let $u=v+\zeta_{v}$ be the decomposition implied by Proposition A. Then, for $t \in\left[0, T_{\delta}\right]$, there exist constants $\nu^{\prime}, C_{1}, C_{2}, C_{3}>0$ such that

$$
\begin{aligned}
& \frac{1}{2} \partial_{t}\left\langle\zeta_{v_{\sigma}}, L_{\epsilon ; v_{\sigma}} \zeta_{v_{\sigma}}\right\rangle \leq-\frac{1}{2} \nu^{\prime}\left\langle\zeta_{v_{\sigma}}, L_{\epsilon ; v_{\sigma}} \zeta_{v_{\sigma}}\right\rangle \\
& +\left\{-C_{1}+C_{2}\left\|\zeta_{v_{\sigma}}\right\|_{H_{v_{\sigma}}^{1}}\left[\left(2+\left\|\zeta_{v_{\sigma}}\right\|_{H_{v_{\sigma}}^{1}}\right)\left(1+\epsilon+\left\|\dot{v}_{\sigma}+P_{v_{\sigma}} \mathcal{E}_{\epsilon}^{\prime}\left(v_{\sigma}\right)\right\|_{H_{v_{\sigma}}^{-s}}\right)-1\right]\right\}\left\|\zeta_{v_{\sigma}}\right\|_{H_{v_{\sigma}}^{2}}^{2} \\
& \quad+\epsilon C_{3}\left(1+\left\|\dot{v}_{\sigma}+P_{v_{\sigma}} \mathcal{E}_{\epsilon}^{\prime}\left(v_{\sigma}\right)\right\|_{H_{v_{\sigma}}^{-s}}+\left\|\zeta_{v_{\sigma}}\right\|_{H_{v_{\sigma}}^{1}}+\left\|\zeta_{v_{\sigma}}\right\|_{H_{v_{\sigma}}^{1}}^{2}\right)\left\|\zeta_{v_{\sigma}}\right\|_{H_{v_{\sigma}}^{1}}
\end{aligned}
$$

Assume that there exists a maximum time $T_{1}$ such that

$$
\left\|\dot{v}_{\sigma}+P_{v_{\sigma}} \mathcal{E}_{\epsilon}^{\prime}\left(v_{\sigma}\right)\right\|_{H_{v_{\sigma}}^{-s}} \leq \epsilon, \quad t \in\left[0, T_{1}\right] .
$$

By Eq. (3.52) and Eq. (3.53) we have in this time interval

$$
\begin{aligned}
& \frac{1}{2} \partial_{t}\left\langle\zeta_{v_{\sigma}}, L_{\epsilon ; v_{\sigma}} \zeta_{v_{\sigma}}\right\rangle \leq\left\{-C_{1}+C_{2}\left\|\zeta_{v_{\sigma}}\right\|_{H_{v_{\sigma}}^{1}}\left[\left(2+\left\|\zeta_{v_{\sigma}}\right\|_{H_{v_{\sigma}}^{1}}\right)(1+2 \epsilon)-1\right]\right\}\left\|\zeta_{v_{\sigma}}\right\|_{H_{v_{\sigma}}^{2}}^{2} \\
& +\epsilon C_{3}\left(1+\epsilon+\left\|\zeta_{v_{\sigma}}\right\|_{H_{v_{\sigma}}^{1}}+\left\|\zeta_{v_{\sigma}}\right\|_{H_{v_{\sigma}}^{1}}^{2}\right)\left\|\zeta_{v_{\sigma}}\right\|_{H_{v_{\sigma}}^{1}}-\frac{1}{2} \nu^{\prime}\left\langle\zeta_{v_{\sigma}}, L_{\epsilon ; v_{\sigma}} \zeta_{v_{\sigma}}\right\rangle .
\end{aligned}
$$

Let $\delta>0$ be such that the decomposition $u=v_{\sigma}(u)+\zeta_{v_{\sigma}}$ implied by Proposition A holds for any $u \in U_{\delta} \subset X^{(n)}$ (see Eq. (3.25) for the definition of $U_{\delta}$ ). The existence of such a $\delta$ is guaranteed by Proposition A. Suppose that there exists a maximum time $T_{2}$ such that 


$$
\left\|\zeta_{v_{\sigma}}\right\|_{H_{v_{\sigma}}^{1}} \leq \min \left\{1, \frac{C_{1}}{10 C_{2}}, \delta\right\}, \quad t \in\left[0, T_{2}\right] .
$$

Set $\tau=\min \left\{T_{1}, T_{2}\right\}$. Then for $t \in[0, \tau]$ we have, for some $\tilde{C}, C>0$,

$$
\frac{1}{2} \partial_{t}\left\langle\zeta_{v_{\sigma}}, L_{\epsilon ; v_{\sigma}} \zeta_{v_{\sigma}}\right\rangle \leq-\frac{1}{2} \nu^{\prime}\left\langle\zeta_{v_{\sigma}}, L_{\epsilon ; v_{\sigma}} \zeta_{v_{\sigma}}\right\rangle-\frac{C_{1}}{2}\left\|\zeta_{v_{\sigma}}\right\|_{H_{v_{\sigma}}^{2}}^{2}+\epsilon \frac{C}{2}\left\|\zeta_{v_{\sigma}}\right\|_{H_{v_{\sigma}}^{1}}
$$

Dropping the negative definite second term on the r.h.s. of Eq. (3.56) and using Corollary $\mathrm{B}$ of the linear stability theorem we get

$$
\frac{1}{2} \partial_{t}\left\langle\zeta_{v_{\sigma}}, L_{\epsilon ; v_{\sigma}} \zeta_{v_{\sigma}}\right\rangle \leq-\frac{1}{2} \nu^{\prime}\left\langle\zeta_{v_{\sigma}}, L_{\epsilon ; v_{\sigma}} \zeta_{v_{\sigma}}\right\rangle+\epsilon \frac{\alpha}{2}\left\langle\zeta_{v_{\sigma}}, L_{\epsilon ; v_{\sigma}} \zeta_{v_{\sigma}}\right\rangle^{1 / 2}
$$

where $\alpha>0$. Set $\left\langle\zeta_{v_{\sigma}}(t), L_{\epsilon ; v_{\sigma}(t)} \zeta_{v_{\sigma}}(t)\right\rangle=f^{2}(t)$, then Eq. (3.57) reads

$$
\frac{d}{d t} f \leq-\nu^{\prime} f+\epsilon \alpha
$$

Let $g(t)$ be a solution of Eq. (3.58) with an equality sign and initial condition $g(0)=$ $f(0)=\left\langle\zeta_{v_{\sigma}}(0), L_{\epsilon ; v_{\sigma}(0)} \zeta_{v_{\sigma}}(0)\right\rangle^{1 / 2}$ i.e.,

$$
\frac{d}{d t} g=-\nu^{\prime} g+\epsilon \alpha
$$

We get

$$
g(t)=f(0) e^{-\nu^{\prime} t}+\frac{\epsilon \alpha}{\nu^{\prime}}\left(1-e^{-\nu^{\prime} t}\right) \leq f(0)+\frac{\epsilon \alpha}{\nu^{\prime}} .
$$

With the help of Lemma F, stated in Section 4, we observe that

$$
\begin{aligned}
f^{2}(0) & =\left\langle\zeta_{v_{\sigma}}(0), L_{\epsilon ; v_{\sigma}(0)} \zeta_{v_{\sigma}}(0)\right\rangle=\left\langle\zeta_{v_{\sigma}}(0), \bar{P}_{v_{\sigma}}(0) L_{\epsilon ; v_{\sigma}(0)} \zeta_{v_{\sigma}}(0)\right\rangle \\
& \leq\left\|\zeta_{v_{\sigma}}(0)\right\|_{H_{v_{\sigma}(0)}^{1}}\left\|\bar{P}_{v_{\sigma}(0)} L_{\epsilon ; v_{\sigma}(0)} \zeta_{v_{\sigma}}(0)\right\|_{H_{v_{\sigma}(0)}^{-1}} \leq C\left\|\zeta_{v_{\sigma}}(0)\right\|_{H_{v_{\sigma}(0)}^{1}}^{2} \leq C^{\prime}\left\|\zeta_{v_{\sigma}}(0)\right\|_{H^{1}}^{2}
\end{aligned}
$$

The validity of the last inequality stems from the fact that $v_{\sigma}(0)$ is some fixed point on $M_{a s}$ and we can estimate the gauge function there.

Now, Proposition A guarantees the existence of a neighborhood $U_{\delta}$ of $M_{a s}$ in which the decomposition property holds. Take $u(0)$ to be an initial data for Eq. (1.1) with $u(0) \in U_{\delta}$. Then we have $\zeta_{v_{\sigma}}(0)=u(0)-v(u(0))$ with $P_{v(u(0))} \zeta_{v_{\sigma}}(0)=0$. Assume furthermore that $u(0)$ is chosen to satisfy the condition that there exists a point $v_{\mathbf{z}_{0} \gamma_{0}}=v_{\sigma_{0}} \in M_{a s}$ such that $\left\|u(0)-v_{\sigma_{0}}\right\|_{X^{(n)}}=\left\|u(0)-v_{\sigma_{0}}\right\|_{H^{1}} \leq c_{0} \epsilon$ for some constant $c_{0}>0$. Under these conditions we have

$$
\left\|\zeta_{v_{\sigma}}(0)\right\|_{H^{1}} \leq\left\|u(0)-v_{\sigma_{0}}\right\|_{H^{1}}+\left\|v_{\sigma_{0}}-v(u(0))\right\|_{H^{1}} \leq c_{0} \epsilon+\left\|v_{\sigma_{0}}-v(u(0))\right\|_{H^{1}}
$$


Denoting $u^{\prime}=v_{\sigma_{0}}$, the solution $v\left(u^{\prime}\right)$ satisfying the orthogonality condition, Eq. (3.26), is simply $v\left(u^{\prime}\right)=u^{\prime}$. We need to show that for $u^{\prime \prime}$ in the vicinity of $u^{\prime}=v_{\sigma_{0}}$ we have

$$
\left\|v\left(u^{\prime}\right)-v\left(u^{\prime \prime}\right)\right\|_{H^{1}} \leq c^{\prime}\left\|u^{\prime}-u^{\prime \prime}\right\|_{H^{1}}
$$

for some constant $c^{\prime}>0$ which may depend only on $\epsilon$, since then we would have $\| v_{\sigma_{0}}-$ $v(u(0))\left\|_{H^{1}} \leq c^{\prime}\right\| v_{\sigma_{0}}-u(0) \|_{H^{1}} \leq c^{\prime} c_{0} \epsilon$. In order to see that Eq. (3.59) holds near $u^{\prime}=v_{\sigma_{0}}$ we first observe that by the implicit function argument in the proof of Proposition A we have

$$
0=D_{u}\left[g\left(u ; M_{d e c}(u)\right)\right]=D_{u} g\left(u ; M_{d e c}(u)\right)+D_{u} M_{d e c}(u) D_{\sigma} g\left(u ; M_{d e c}(u)\right)
$$

and hence

$$
D_{u} M_{d e c}(u)=-\left[D_{\sigma} g\left(u ; M_{d e c}(u)\right)\right]^{-1} D_{u} g\left(u ; M_{d e c}(u)\right) .
$$

It follows that $\left\|D_{u} M_{d e c}\left(v_{\sigma_{0}}\right)\right\|_{X^{(n)} \rightarrow R^{2} \times H^{2}} \leq C$; since Eq. (3.30) (recall that $K_{\sigma}$ is uniformly invertible on $\left.M_{a s}\right)$ and the definition of $g(u ; \sigma)$ in Eq. (3.28) imply, respectively, that the two mappings $\left\|\left[D_{\sigma} g\left(v_{\sigma_{0}} ; \sigma_{0}\right)\right]^{-1}\right\|_{R^{2} \times L^{2} \rightarrow R^{2} \times H^{2}}$ and $\left\|D_{u} g\left(v_{\sigma_{0}} ; \sigma_{0}\right)\right\|_{X^{(n)} \rightarrow R^{2} \times L^{2}}$ are uniformly bounded on $M_{a s}$. This observation leads to the following bound

$$
\begin{aligned}
\left\|D_{u} v\left(v_{\sigma_{0}}\right)\right\|_{X^{(n)} \rightarrow X^{(n)}} & =\left\|D_{u}\left[\beta\left(M_{d e c}\left(v_{\sigma_{0}}\right)\right)\right]\right\|_{X^{(n)} \rightarrow X^{(n)}} \\
& \left.\leq\left\|\left(D_{\sigma} v_{\sigma}\right)_{\sigma_{0}}\right\|_{R^{2} \times H^{2} \rightarrow X^{(n)}} \| D_{u} M_{d e c}\left(v_{\sigma_{0}}\right)\right) \|_{X^{(n)} \rightarrow R^{2} \times H^{2}} \leq C
\end{aligned}
$$

and we conclude that, for $c_{0}=c_{0}(\epsilon)$ small enough, if $\left\|u(0)-v_{\sigma_{0}}\right\|_{H^{1}} \leq c_{0} \epsilon$ then $f(0) \leq c \epsilon$ for some constant $c=c(\epsilon)>0$. Hence, in the time interval $t \in[0, \tau]$, we have

$$
\left\|\zeta_{v}\right\|_{H^{1}} \leq \nu^{-1}\left\langle\zeta_{v}, L_{\epsilon ; v} \zeta_{v}\right\rangle^{1 / 2}=\nu^{-1} f(t) \leq \nu^{-1} g(t) \leq C \epsilon .
$$

In order to close the proof of Proposition $\mathrm{C}$ we need an estimate on the time derivatives $\dot{\mathbf{z}}$ and $\dot{\tilde{\gamma}}$ appearing in the last term on the r.h.s of Eq. (3.40). The following lemma is proved in Subsection 5.1:

Lemma D: For $s>0$ we have the following estimate for the parametric equations of motion

$$
\left|a_{n} \dot{\mathbf{z}}+\partial_{\mathbf{z}}^{A} \mathcal{E}_{\epsilon}\left(v_{\sigma}\right)\right|+\|\dot{\tilde{\gamma}}\|_{H^{1-s}} \leq C\left\|\dot{v}_{\sigma}+P_{v_{\sigma}} \mathcal{E}_{\epsilon}^{\prime}\left(v_{\sigma}\right)\right\|_{H_{v_{\sigma}}^{-s}}
$$

where $\dot{\tilde{\gamma}}$ is defined in Eq. (3.14).

Since $\partial_{z_{i}}^{A} \mathcal{E}_{\epsilon}\left(v_{\sigma}\right)=o(\epsilon)$ (see Eq. (3.67) below) we get that for $0<s<1$

$|\dot{\mathbf{z}}|+\|\dot{\tilde{\gamma}}\|_{2} \leq a_{n}^{-1}\left(\left|a_{n} \dot{\mathbf{z}}+\partial_{\mathbf{z}}^{A} \mathcal{E}_{\epsilon}\left(v_{\sigma}\right)\right|+\left|\partial_{\mathbf{z}}^{A} \mathcal{E}_{\epsilon}\left(v_{\sigma}\right)\right|\right)+\|\dot{\tilde{\gamma}}\|_{H^{1-s}} \leq C\left(\left\|\dot{v}_{\sigma}+P_{v_{\sigma}} \mathcal{E}_{\epsilon}^{\prime}\left(v_{\sigma}\right)\right\|_{H_{v_{\sigma}}^{-s}}+\epsilon\right)$ 
then, under the assumption made in Eq. (3.53), we have

$$
|\dot{\mathbf{z}}|+\|\dot{\tilde{\gamma}}\|_{2} \leq \epsilon C, \quad t \in[0, \tau] .
$$

Eq. (3.40), Eq. (3.60) and Eq. (3.63) then imply that for $t \in[0, \tau]$ the following inequality is valid

$\left\|\dot{v}_{\sigma}+P_{v_{\sigma}} \mathcal{E}_{\epsilon}^{\prime}\left(v_{\sigma}\right)\right\|_{H_{v_{\sigma}}^{-s}} \leq C\left[\epsilon\left\|\zeta_{v_{\sigma}}\right\|_{H_{v_{\sigma}}^{1}}+\left\|\zeta_{v_{\sigma}}\right\|_{H_{v_{\sigma}}^{1}}^{2}+\left\|\zeta_{v_{\sigma}}\right\|_{H_{v_{\sigma}}^{1}}^{3}+\left(|\dot{\mathbf{z}}|+\|\dot{\tilde{\gamma}}\|_{2}\right)\left\|\zeta_{v_{\sigma}}\right\|_{H_{v_{\sigma}}^{1}}\right] \leq C \epsilon^{2}$

The definition of $\tau$ implies that at $t=\tau$ at least one of the conditions, Eq. (3.53) or Eq. (3.55), is no longer valid. However, we can choose $\epsilon_{0}$ in such a way that for $0<\epsilon<\epsilon_{0}$ this conclusion will stand in contradiction with the results, Eq. (3.60) and Eq. (3.64). Hence $\tau=\infty$ and the proof of Proposition $\mathrm{C}$ is complete.

Step 4 - Effective equations for the parameters

We derive the appropriate equations for the variables $\mathbf{z}$ and $\gamma$, parametrizing the manifold $M_{a s}$. Thus, solutions of Eq. (1.1) with an initial condition close enough to $M_{a s}$ induce, up to a small, controlable error, an effective dynamics on $M_{a s}$ in terms of equations of motion for the vortex center and gauge function in the presence of the potential $W$.

The effective equations of motion for the parameters $\mathbf{z}$ and $\tilde{\gamma}$ are derived using Eq. (3.61) and proposition C. For $\tilde{\gamma}$ we have simply

$$
\|\dot{\tilde{\gamma}}\|_{H^{1-s}} \leq C \epsilon^{2}
$$

and for $\mathbf{z}$ we get

$$
\left|a_{n} \dot{\mathbf{z}}+\partial_{\mathbf{z}}^{A} \mathcal{E}_{\epsilon}\left(v_{\sigma}\right)\right| \leq C \epsilon^{2}
$$

Since $\mathcal{E}_{\epsilon}\left(v_{\sigma}\right)=\mathcal{E}_{\text {int }}\left(v_{\sigma}\right)=W_{\text {int }}^{(n)}(\mathbf{z})$, where

$$
W_{i n t}^{(n)}(\mathbf{z}) \equiv \epsilon \int d^{2} x W(\mathbf{x})\left|\psi^{(n)}(\mathbf{x}-\mathbf{z})\right|^{2}
$$

does not depend on the vector potential $A$, we get finally

$$
\left|a_{n} \dot{\mathbf{z}}+\nabla_{\mathbf{z}} W_{\text {int }}(\mathbf{z})\right| \leq C \epsilon^{2}
$$

This derivation of the parametric equations of motion completes the proof of Theorem A.

\section{Proof of Proposition D}

We calculate 


$$
\begin{aligned}
& \frac{1}{2} \partial_{t}\left\langle\zeta_{v}, L_{\epsilon, v} \zeta_{v}\right\rangle=\partial_{t}\left(\mathcal{E}_{\epsilon}(u)-\mathcal{E}_{\epsilon}(v)-\left\langle\mathcal{E}_{\epsilon}^{\prime}(v), \zeta_{v}\right\rangle-R_{v}\left(\zeta_{v}\right)\right)= \\
= & \left\langle\mathcal{E}_{\epsilon}^{\prime}(u), \dot{u}\right\rangle-\left\langle\mathcal{E}_{\epsilon}^{\prime}(v), \dot{v}\right\rangle-\left\langle\mathcal{E}_{\epsilon}^{\prime \prime}(v) \dot{v}, \zeta_{v}\right\rangle-\left\langle\mathcal{E}_{\epsilon}^{\prime}(v), \dot{\zeta}_{v}\right\rangle-\partial_{t}\left[R_{v}\left(\zeta_{v}\right)\right]= \\
= & \left\langle\mathcal{E}_{\epsilon}^{\prime}(u)-\mathcal{E}_{\epsilon}^{\prime}(v), \dot{u}\right\rangle-\left\langle L_{\epsilon, v} \dot{v}, \zeta_{v}\right\rangle-\partial_{t}\left[R_{v}\left(\zeta_{v}\right)\right]= \\
= & -\left\langle\mathcal{E}_{\epsilon}^{\prime}(u)-\mathcal{E}_{\epsilon}^{\prime}(v), \mathcal{E}_{\epsilon}^{\prime}(u)\right\rangle-\left\langle L_{\epsilon, v} \dot{v}, \zeta_{v}\right\rangle-\partial_{t}\left[R_{v}\left(\zeta_{v}\right)\right] \\
= & -\left\langle\mathcal{E}_{\epsilon}^{\prime}(u)-\mathcal{E}_{\epsilon}^{\prime}(v), \bar{P}_{v} \mathcal{E}_{\epsilon}^{\prime}(u)\right\rangle+\left\langle\mathcal{E}_{\epsilon}^{\prime}(v)-\mathcal{E}_{\epsilon}^{\prime}(u), P_{v} \mathcal{E}_{\epsilon}^{\prime}(u)\right\rangle-\left\langle L_{\epsilon, v} \dot{v}, \zeta_{v}\right\rangle-\partial_{t}\left[R_{v}\left(\zeta_{v}\right)\right]
\end{aligned}
$$

where the energy remainder term $R_{v}(\zeta)$ is given in Appendix A, Eq. (A.5). Next we define the quantity $\dot{R}_{v}\left(\zeta_{v}\right)$ by

$$
\partial_{t}\left[R_{v}\left(\zeta_{v}\right)\right]=\dot{R}_{v}\left(\zeta_{v}\right)+\left\langle N_{v}\left(\zeta_{v}\right), \dot{\zeta}_{v}\right\rangle .
$$

Consider the equation of motion for the error term $\zeta_{v}$. This equation is obtained by projecting Eq. (1.12) on $\left(T_{v} M_{a s}\right)^{\perp}$. We have

$$
\dot{\zeta}_{v}=-\bar{P}_{v} \mathcal{E}_{\epsilon}^{\prime}(u)-\dot{P}_{v} \zeta_{v} .
$$

From Eq. (4.2) and Eq. (4.3) we get

$$
\partial_{t}\left[R_{v}\left(\zeta_{v}\right)\right]=\dot{R}_{v}\left(\zeta_{v}\right)-\left\langle N_{v}\left(\zeta_{v}\right), \bar{P}_{v} \mathcal{E}_{\epsilon}^{\prime}(u)\right\rangle-\left\langle N_{v}\left(\zeta_{v}\right), \dot{P}_{v} \zeta_{v}\right\rangle .
$$

Inserting Eq. (4.4) into Eq. (4.1) we obtain the following inequality

$$
\begin{aligned}
\frac{1}{2} \partial_{t}\left\langle\zeta_{v}, L_{\epsilon, v} \zeta_{v}\right\rangle=-\left\langle L_{\epsilon, v} \zeta_{v}, \bar{P}_{v} \mathcal{E}_{\epsilon}^{\prime}(u)\right\rangle & -\left\langle\mathcal{E}_{\epsilon}^{\prime}(u)-\mathcal{E}_{\epsilon}^{\prime}(v), P_{v} \mathcal{E}_{\epsilon}^{\prime}(u)\right\rangle \\
& -\left\langle L_{\epsilon, v} \dot{v}, \zeta_{v}\right\rangle-\dot{R}_{v}\left(\zeta_{v}\right)+\left\langle N_{v}\left(\zeta_{v}\right), \dot{P}_{v} \zeta_{v}\right\rangle= \\
\leq-\left\langle L_{\epsilon, v} \zeta_{v}, \bar{P}_{v} \mathcal{E}_{\epsilon}^{\prime}(u)\right\rangle & -\left\langle\mathcal{E}_{\epsilon}^{\prime}(u)-\mathcal{E}_{\epsilon}^{\prime}(v), P_{v} \mathcal{E}_{\epsilon}^{\prime}(v)\right\rangle \\
& -\left\langle L_{\epsilon, v} \dot{v}, \zeta_{v}\right\rangle-\dot{R}_{v}\left(\zeta_{v}\right)+\left\langle N_{v}\left(\zeta_{v}\right), \dot{P}_{v} \zeta_{v}\right\rangle= \\
=- & \left\langle\bar{P}_{v} L_{\epsilon, v} \zeta_{v}, \bar{P}_{v} L_{\epsilon, v} \zeta_{v}\right\rangle \\
- & +\left\langle L_{\epsilon, v} \zeta_{v}, \bar{P}_{v}\left(\mathcal{E}_{\epsilon}^{\prime}(v)+N_{v}\left(\zeta_{v}\right)\right)\right\rangle \\
-\left\langle L_{\epsilon ; v} \zeta_{v}, \dot{v}+P_{v} \mathcal{E}_{\epsilon}^{\prime}(v)\right\rangle & -\left\langle N_{v}\left(\zeta_{v}\right), P_{v} \mathcal{E}_{\epsilon}^{\prime}(v)\right\rangle-\dot{R}_{v}\left(\zeta_{v}\right)+\left\langle N_{v}\left(\zeta_{v}\right), \dot{P}_{v} \zeta_{v}\right\rangle
\end{aligned}
$$

and so

$$
\begin{aligned}
& \frac{1}{2} \partial_{t}\left\langle\zeta_{v}, L_{\epsilon ; v} \zeta_{v}\right\rangle \leq-\left\langle\bar{P}_{v} L_{\epsilon, v} \zeta_{v}, \bar{P}_{v} L_{\epsilon, v} \zeta_{v}\right\rangle+\left|\left\langle L_{\epsilon, v} \zeta_{v}, \bar{P}_{v}\left(\mathcal{E}_{\epsilon}^{\prime}(v)+N_{v}\left(\zeta_{v}\right)\right)\right\rangle\right| \\
& +\left|\left\langle L_{\epsilon ; v} \zeta_{v}, \dot{v}+P_{v} \mathcal{E}_{\epsilon}^{\prime}(v)\right\rangle\right|+\left|\left\langle N_{v}\left(\zeta_{v}\right), P_{v} \mathcal{E}_{\epsilon}^{\prime}(v)\right\rangle\right|+\left|\dot{R}_{v}\left(\zeta_{v}\right)\right|+\left|\left\langle N_{v}\left(\zeta_{v}\right), \dot{P}_{v} \zeta_{v}\right\rangle\right|
\end{aligned}
$$

In Subsection 5.2 we establish, via straightforward calculations, the validity of the following estimates

Lemma E: For $\zeta \in T_{v} X^{(n)}$ we have 


$$
\left\|N_{v_{\sigma}}(\zeta)\right\|_{2} \leq C\|\zeta\|_{H_{v_{\sigma}}^{2}}\left(\|\zeta\|_{H_{v_{\sigma}}^{1}}+\|\zeta\|_{H_{v_{\sigma}}^{1}}^{2}\right)
$$

and

Lemma F: For $\zeta \in T_{v} X^{(n)}$ the following inequalities hold

$$
\left\|\bar{P}_{v_{\sigma}} L_{\epsilon ; v_{\sigma}} \zeta\right\|_{H_{v_{\sigma}}^{-1}} \leq C\|\zeta\|_{H_{v_{\sigma}}^{1}}, \quad\left\|\bar{P}_{v_{\sigma}} L_{\epsilon ; v_{\sigma}} \zeta\right\|_{2} \leq C\|\zeta\|_{H_{v_{\sigma}}^{2}} .
$$

Eq. (4.7), Eq. (4.8) and the easily obtainable inequality

$$
\left\|\mathcal{E}_{\epsilon}^{\prime}\left(v_{\sigma}\right)\right\|_{H_{v_{\sigma}}^{1}}=\epsilon\left\|W \psi_{\mathbf{z} 0}\right\|_{H^{1}} \leq \epsilon C
$$

result in the following estimates:

$$
\left|\left\langle\bar{P}_{v_{\sigma}} L_{\epsilon ; v_{\sigma}} \zeta_{v_{\sigma}}, \mathcal{E}_{\epsilon}^{\prime}\left(v_{\sigma}\right)\right\rangle\right| \leq\left\|\bar{P}_{v_{\sigma}} L_{\epsilon, v_{\sigma}} \zeta_{v_{\sigma}}\right\|_{H_{v_{\sigma}}^{-1}}\left\|\mathcal{E}_{\epsilon}^{\prime}\left(v_{\sigma}\right)\right\|_{H_{v_{\sigma}}^{1}} \leq \epsilon C\left\|\zeta_{v_{\sigma}}\right\|_{H_{v_{\sigma}}^{1}}
$$

and

$$
\begin{aligned}
\left|\left\langle\bar{P}_{v_{\sigma}} L_{\epsilon ; v_{\sigma}} \zeta_{v_{\sigma}}, N_{v_{\sigma}}\left(\zeta_{v_{\sigma}}\right)\right\rangle\right| & \leq\left\|\bar{P}_{v_{\sigma}} L_{\epsilon ; v_{\sigma}} \zeta_{v_{\sigma}}\right\|_{2}\left\|N_{v_{\sigma}}\left(\zeta_{v_{\sigma}}\right)\right\|_{2} \\
& \leq C\left\|\zeta_{v_{\sigma}}\right\|_{H_{v_{\sigma}}^{2}}^{2}\left(\left\|\zeta_{v_{\sigma}}\right\|_{H_{v_{\sigma}}^{1}}+\left\|\zeta_{v_{\sigma}}\right\|_{H_{v_{\sigma}}^{1}}^{2}\right) .
\end{aligned}
$$

Furthermore, we have the two estimates

$$
\begin{aligned}
&\left|\left\langle L_{\epsilon ; v_{\sigma}} \zeta_{v_{\sigma}}, \dot{v}_{\sigma}+P_{v_{\sigma}} \mathcal{E}_{\epsilon}^{\prime}\left(v_{\sigma}\right)\right\rangle\right|=\left|\left\langle P_{v_{\sigma}} L_{\epsilon ; v_{\sigma}} \zeta_{v_{\sigma}}, \dot{v}_{\sigma}+P_{v_{\sigma}} \mathcal{E}_{\epsilon}^{\prime}\left(v_{\sigma}\right)\right\rangle\right| \\
& \quad \leq\left\|P_{v_{\sigma}} L_{\epsilon ; v_{\sigma}} \zeta_{v_{\sigma}}\right\|_{H_{v_{\sigma}}^{1}}\left\|\dot{v}_{\sigma}+P_{v_{\sigma}} \mathcal{E}_{\epsilon}^{\prime}\left(v_{\sigma}\right)\right\|_{H_{v_{\sigma}}^{-1}} \leq \epsilon C\left\|\zeta_{v_{\sigma}}\right\|_{H_{v_{\sigma}}^{1}}\left\|\dot{v}_{\sigma}+P_{v_{\sigma}} \mathcal{E}_{\epsilon}^{\prime}\left(v_{\sigma}\right)\right\|_{H_{v_{\sigma}}^{-s}}
\end{aligned}
$$

(see the proof of Lemma A) and

$$
\left|\left\langle N_{v_{\sigma}}\left(\zeta_{v_{\sigma}}\right), P_{v_{\sigma}} \mathcal{E}_{\epsilon}^{\prime}\left(v_{\sigma}\right)\right\rangle\right| \leq\left\|P_{v_{\sigma}} N_{v_{\sigma}}\left(\zeta_{v_{\sigma}}\right)\right\|_{H_{v_{\sigma}}^{-1}}\left\|\mathcal{E}_{\epsilon}^{\prime}\left(v_{\sigma}\right)\right\|_{H_{v_{\sigma}}^{1}} \leq \epsilon C\left(\left\|\zeta_{v_{\sigma}}\right\|_{H_{v_{\sigma}}^{1}}^{2}+\left\|\zeta_{v_{\sigma}}\right\|_{H_{v_{\sigma}}^{1}}^{3}\right)
$$

where in Eq. (4.13) use has been made of Eq. (4.9) and Lemma B.

In order to obtain a bound on the term $\dot{R}_{v}\left(\zeta_{v}\right)$ we note that Eq. (4.2) implies

$$
\dot{R}_{v}(\zeta)=\left(\partial_{t} R_{v}(\zeta)\right)_{\zeta=\text { const. }}=\left\langle\dot{v}, \partial_{v} R_{v}(\zeta)\right\rangle
$$

hence, we have

$$
\begin{aligned}
\left|\dot{R}_{v_{\sigma}}\left(\zeta_{v_{\sigma}}\right)\right| & =\left|\left\langle\dot{v}_{\sigma}, \partial_{v_{\sigma}} R_{v_{\sigma}}\left(\zeta_{v_{\sigma}}\right)\right\rangle\right| \\
& \leq\left(\left\|\dot{v}_{\sigma}+P_{v_{\sigma}} \mathcal{E}_{\epsilon}^{\prime}\left(v_{\sigma}\right)\right\|_{H_{v_{\sigma}}^{-s}}+\left\|P_{v_{\sigma}} \mathcal{E}_{\epsilon}^{\prime}\left(v_{\sigma}\right)\right\|_{H_{v_{\sigma}}^{-s}}\right)\left\|\partial_{v_{\sigma}} R_{v_{\sigma}}\left(\zeta_{v_{\sigma}}\right)\right\|_{H_{v_{\sigma}}^{s}} .
\end{aligned}
$$


The following estimate is proved in Subsection 5.2:

Lemma G: For any $\zeta \in T_{v_{\sigma}} X$ we have

$$
\left\|\partial_{v_{\sigma}} R_{v_{\sigma}}(\zeta)\right\|_{H_{v_{\sigma}}^{1}} \leq C\|\zeta\|_{H_{v_{\sigma}}^{2}}^{2}\|\zeta\|_{H_{v_{\sigma}}^{1}} .
$$

Lemma $\mathrm{G}$ and Eq. (4.9) then imply the following bound for $0<s<1$

$$
\left|\dot{R}_{v_{\sigma}}\left(\zeta_{v_{\sigma}}\right)\right| \leq C\left(\left\|\dot{v}_{\sigma}+P_{v_{\sigma}} \mathcal{E}_{\epsilon}^{\prime}\left(v_{\sigma}\right)\right\|_{H_{v_{\sigma}}^{-s}}+\epsilon\right)\left\|\zeta_{v_{\sigma}}\right\|_{H_{v_{\sigma}}^{2}}^{2}\left\|\zeta_{v_{\sigma}}\right\|_{H_{v_{\sigma}}^{1}} .
$$

With Eq. (4.10), (4.11), (4.12), (4.13) and (4.17) providing appropriate bounds on the corresponding terms in Eq. (4.6) we get

$$
\begin{aligned}
& \frac{1}{2} \partial_{t}\left\langle\zeta_{v_{\sigma}}, L_{\epsilon ; v_{\sigma}} \zeta_{v_{\sigma}}\right\rangle \leq-\left\langle\bar{P}_{v_{\sigma}} L_{\epsilon ; v_{\sigma}} \zeta_{v_{\sigma}}, \bar{P}_{v_{\sigma}} L_{\epsilon ; v_{\sigma}} \zeta_{v_{\sigma}}\right\rangle \\
& +C\left(1+\epsilon+\left\|\zeta_{v_{\sigma}}\right\|_{H_{v_{\sigma}}^{1}}+\left\|\dot{v}_{\sigma}+P_{v_{\sigma}} \mathcal{E}_{\epsilon}^{\prime}\left(v_{\sigma}\right)\right\|_{H_{v_{\sigma}}^{-s}}\right)\left\|\zeta_{v_{\sigma}}\right\|_{H_{v_{\sigma}}^{1}}\left\|\zeta_{v_{\sigma}}\right\|_{H_{v_{\sigma}}^{2}}^{2} \\
& +\epsilon C\left(1+\left\|\dot{v}_{\sigma}+P_{v_{\sigma}} \mathcal{E}_{\epsilon}^{\prime}\left(v_{\sigma}\right)\right\|_{H_{v_{\sigma}}^{-s}}+\left\|\zeta_{v_{\sigma}}\right\|_{H_{v_{\sigma}}^{1}}+\left\|\zeta_{v_{\sigma}}\right\|_{H_{v_{\sigma}}^{1}}^{2}\right)\left\|\zeta_{v_{\sigma}}\right\|_{H_{v_{\sigma}}^{1}}+\mid\left\langle N_{v_{\sigma}}\left(\zeta_{v_{\sigma}}\right), \dot{P}_{v_{\sigma}} \zeta_{v_{\sigma}}\right\rangle .
\end{aligned}
$$

We still need to estimate the last term on the r.h.s. of Eq. (4.18). Using Lemma C and Eq. (3.62) we obtain

$$
\left\|\dot{P}_{v_{\sigma}} \zeta_{v_{\sigma}}\right\|_{2} \leq C\left(\left\|\dot{v}_{\sigma}+P_{v_{\sigma}} \mathcal{E}_{\epsilon}^{\prime}\left(v_{\sigma}\right)\right\|_{H_{v_{\sigma}}^{-s}}+\epsilon\right)\left\|\zeta_{v_{\sigma}}\right\|_{H_{v_{\sigma}}^{1}} .
$$

With the help of Lemma E and Eq. (4.19) we can provide the appropriate bound in the form

$$
\begin{aligned}
\left|\left\langle N_{v_{\sigma}}\left(\zeta_{v_{\sigma}}\right), \dot{P}_{v_{\sigma}} \zeta_{v_{\sigma}}\right\rangle\right| & \leq\left\|N_{v_{\sigma}}\left(\zeta_{v_{\sigma}}\right)\right\|_{2}\left\|\dot{P}_{v_{\sigma}} \zeta_{v_{\sigma}}\right\|_{2} \\
& \leq C\left(\left\|\dot{v}_{\sigma}+P_{v_{\sigma}} \mathcal{E}_{\epsilon}^{\prime}\left(v_{\sigma}\right)\right\|_{H_{v_{\sigma}}^{-s}}+\epsilon\right)\left(1+\left\|\zeta_{v_{\sigma}}\right\|_{H_{v_{\sigma}}^{1}}\right)\left\|\zeta_{v_{\sigma}}\right\|_{H_{v_{\sigma}}^{1}}\left\|\zeta_{v_{\sigma}}\right\|_{H_{v_{\sigma}}^{2}}^{2}
\end{aligned}
$$

Inserting the bound obtained in Eq. (4.20) into the r.h.s. of Eq. (4.18) we get

$$
\begin{aligned}
& \frac{1}{2} \partial_{t}\left\langle\zeta_{v_{\sigma}}, L_{\epsilon ; v_{\sigma}} \zeta_{v_{\sigma}}\right\rangle \leq-\left\langle\bar{P}_{v_{\sigma}} L_{\epsilon ; v_{\sigma}} \zeta_{v_{\sigma}}, \bar{P}_{v_{\sigma}} L_{\epsilon ; v_{\sigma}} \zeta_{v_{\sigma}}\right\rangle \\
& +C_{2}\left[\left(2+\left\|\zeta_{v_{\sigma}}\right\|_{H_{v_{\sigma}}^{1}}\right)\left(1+\epsilon+\left\|\dot{v}_{\sigma}+P_{v_{\sigma}} \mathcal{E}_{\epsilon}^{\prime}\left(v_{\sigma}\right)\right\|_{H_{v_{\sigma}}^{-s}}\right)-1\right]\left\|\zeta_{v_{\sigma}}\right\|_{H_{v_{\sigma}}^{1}}\left\|\zeta_{v_{\sigma}}\right\|_{H_{v_{\sigma}}^{2}}^{2} \\
& +\epsilon C_{3}\left(1+\left\|\dot{v}_{\sigma}+P_{v_{\sigma}} \mathcal{E}_{\epsilon}^{\prime}\left(v_{\sigma}\right)\right\|_{H_{v_{\sigma}}^{-s}}+\left\|\zeta_{v_{\sigma}}\right\|_{H_{v_{\sigma}}^{1}}+\left\|\zeta_{v_{\sigma}}\right\|_{H_{v_{\sigma}}^{1}}^{2}\right)\left\|\zeta_{v_{\sigma}}\right\|_{H_{v_{\sigma}}^{1}} .
\end{aligned}
$$

The first term on the r.h.s. of Eq. (4.21) is handled by utilizing the linear stability properties of the Ginzburg-Landau equations. The linear stability theorem is used in Subsection 5.2 in the proof of the following Lemma 
Lemma H: For $\zeta_{v} \in T_{v} X^{(n)}$ orthogonal to $T_{v} M_{a s}$, that is for $P_{v} \zeta_{v}=0$, we have

$$
\left\langle\bar{P}_{v_{\sigma}} L_{\epsilon ; v_{\sigma}} \zeta_{v_{\sigma}}, \bar{P}_{v_{\sigma}} L_{\epsilon ; v_{\sigma}} \zeta_{v_{\sigma}}\right\rangle \geq 2 C_{1}\left\|\zeta_{v_{\sigma}}\right\|_{H_{v_{\sigma}}^{2}}^{2}
$$

(the choice of the form of the constant here is purely for reasons of convenience).

We will also need an additional lemma which is also proved in Subsection 5.2:

Lemma I: For $\zeta \in T_{v} X^{(n)}$ with $\zeta_{v} \in\left(T_{v} M_{a s}\right)^{\perp}$ we have

$$
\left\langle\bar{P}_{v} L_{\epsilon ; v} \zeta_{v}, \bar{P}_{v} L_{\epsilon ; v} \zeta_{v}\right\rangle \geq \nu^{\prime}\left\langle\zeta_{v}, L_{\epsilon ; v} \zeta_{v}\right\rangle
$$

Eq. (4.21), Lemma H and Lemma I imply that

$$
\begin{aligned}
& \frac{1}{2} \partial_{t}\left\langle\zeta_{v_{\sigma}}, L_{\epsilon ; v_{\sigma}} \zeta_{v_{\sigma}}\right\rangle \leq-\frac{1}{2} \nu^{\prime}\left\langle\zeta_{v_{\sigma}}, L_{\epsilon ; v_{\sigma}} \zeta_{v_{\sigma}}\right\rangle \\
& +\left\{-C_{1}+C_{2}\left\|\zeta_{v_{\sigma}}\right\|_{H_{v_{\sigma}}^{1}}\left[\left(2+\left\|\zeta_{v_{\sigma}}\right\|_{H_{v_{\sigma}}^{1}}\right)\left(1+\epsilon+\left\|\dot{v}_{\sigma}+P_{v_{\sigma}} \mathcal{E}_{\epsilon}^{\prime}\left(v_{\sigma}\right)\right\|_{H_{v_{\sigma}}^{-s}}\right)-1\right]\right\}\left\|\zeta_{v_{\sigma}}\right\|_{H_{v_{\sigma}}^{2}}^{2} \\
& +\epsilon C_{3}\left(1+\left\|\dot{v}_{\sigma}+P_{v_{\sigma}} \mathcal{E}_{\epsilon}^{\prime}\left(v_{\sigma}\right)\right\|_{H_{v_{\sigma}}^{-s}}+\left\|\zeta_{v_{\sigma}}\right\|_{H_{v_{\sigma}}^{1}}+\left\|\zeta_{v_{\sigma}}\right\|_{H_{v_{\sigma}}^{1}}^{2}\right)\left\|\zeta_{v_{\sigma}}\right\|_{H_{v_{\sigma}}^{1}} \cdot
\end{aligned}
$$

Eq. (4.24) is the statement of proposition D

\section{Proofs of technical results}

\subsection{Proofs of Lemma C and Lemma D}

\section{Proof of Lemma C:}

The starting point to the proof of Lemma $\mathrm{C}$ is the explicit expression for the projection $P_{v_{\sigma}}$ given in Eq. (3.12). Taking the time derivative of $P_{v_{\sigma}}$ and applying the resulting operator to a fixed vector $\zeta \in T_{v_{\sigma}} X^{(n)}$ satisfying the condition $P_{v_{\sigma}} \zeta=0$ we get

$$
\dot{P}_{v_{\sigma}} \zeta=a_{n}^{-2} \sum_{i=1,2} T_{i}^{\sigma} \partial_{t}\left\langle T_{i}^{\sigma}, \zeta\right\rangle+\int d^{2} x G_{\delta(x)}^{\sigma} K_{\sigma}^{-1}(x, y) \partial_{t}\left\langle G_{\delta(y)}^{\sigma}, \zeta\right\rangle
$$

Eq. (5.1) enable us to obtain the $L^{2}$ norm of $\dot{P}_{v} \zeta$

$$
\begin{aligned}
\left\|\dot{P}_{v_{\sigma}} \zeta\right\|_{2}^{2} & =a_{n}^{-2} \sum_{i=1,2}\left|\left\langle\partial_{t} T_{i}^{\sigma}, \zeta\right\rangle\right|^{2} \\
& +\int d^{2} x \int d^{2} y \overline{\partial_{t}\left\langle G_{\delta(x)}^{\sigma}, \zeta\right\rangle} K_{\sigma}^{-1}(x, y) \partial_{t}\left\langle G_{\delta(y)}^{\sigma}, \zeta\right\rangle
\end{aligned}
$$
by

Consider the first term on the r.h.s. of Eq. (5.2). The time derivative of $T_{i}^{\sigma}$ is given 


$$
\partial_{t} T_{i}^{\sigma}=i \dot{\tilde{\gamma}}\left(\begin{array}{c}
\left(\nabla_{A_{\sigma}} \psi_{\sigma}\right)_{i} \\
0
\end{array}\right)+\Sigma_{j}\left(-\dot{z}_{j}\right)\left(\nabla_{A_{\sigma}} T_{i}^{\sigma}\right)_{j}
$$

where

$$
\left(\nabla_{A_{\sigma}} T_{i}^{\sigma}\right)_{j}=\left(\begin{array}{c}
\left(\nabla_{A_{\sigma}}\left(\nabla_{A_{\sigma}} \psi_{\sigma}\right)_{i}\right)_{j} \\
\partial_{j}\left(\nabla \times A_{\sigma}\right) \hat{e}_{i}
\end{array}\right)
$$

Thus we arrive at the following estimate

$$
\begin{aligned}
\left\|\partial_{t} T_{i}^{\sigma}\right\|_{2} & \leq\left\|\dot{\tilde{\gamma}}\left(\nabla_{A_{\sigma}} \psi_{\sigma}\right)_{i}\right\|_{2}+\Sigma_{j}\left|\dot{z}_{j}\right|\left\|\left(\nabla_{A_{\sigma}} T_{i}^{\sigma}\right)_{j}\right\|_{2} \\
& \leq\|\dot{\tilde{\gamma}}\|_{2}\left\|\left(\nabla_{A_{\sigma}} \psi_{\sigma}\right)_{i}\right\|_{\infty}+\Sigma_{j}\left|\dot{z}_{j}\right|\left\|\left(\nabla_{A_{\sigma}} T_{i}^{\sigma}\right)_{j}\right\|_{2}
\end{aligned} .
$$

The norms of the two vectors appearing on the r.h.s. of Eq. (5.5) are gauge and translation invariant, hence they are constant at all points of $M_{a s}$. We conclude that the following inequality is valid

$$
\left\|\partial_{t} T_{i}^{\sigma}\right\|_{2} \leq C\left(\|\dot{\tilde{\gamma}}\|_{2}+\Sigma_{i}\left|\dot{z}_{i}\right|\right)
$$

and so

$$
\begin{aligned}
\left|\left\langle\partial_{t} T_{i}^{\sigma}, \zeta\right\rangle\right| & \leq\left\|\partial_{t} T_{i}^{\sigma}\right\|_{2}\|\zeta\|_{2} \leq C\left(\|\dot{\tilde{\gamma}}\|_{2}+\sum_{i}\left|\dot{z}_{i}\right|\right)\left\|G_{-\gamma} \zeta\right\|_{2} \\
& \leq C\left(\|\dot{\tilde{\gamma}}\|_{2}+\sum_{i}\left|\dot{z}_{i}\right|\right)\left\|G_{-\gamma} \zeta\right\|_{H^{1}}=C\left(\|\dot{\tilde{\gamma}}\|_{2}+\sum_{i}\left|\dot{z}_{i}\right|\right)\|\zeta\|_{H_{v_{\sigma}}^{1}} .
\end{aligned}
$$

Consider now the second term on the r.h.s. of Eq. (5.2). Let $\zeta=(\xi, F) \in T_{v} X^{(n)}$, the time derivative $\partial_{t}\left\langle G_{\chi}^{\sigma}, \zeta\right\rangle$ is given by (note that $\zeta$ is a fixed vector)

$$
\begin{aligned}
\partial_{t}\left\langle G_{\chi}^{\sigma}, \zeta\right\rangle & =\partial_{t}\left\langle\int d^{2} x \chi(x) G_{\delta(x)}^{\sigma}, \zeta\right\rangle=\int d^{2} x \chi(x) \partial_{t}\left\langle G_{\delta(x)}^{\sigma}, \zeta\right\rangle= \\
& =\partial_{t} \int d^{2} x\left(\operatorname{Im}\left[\chi \bar{\psi}_{\sigma} \xi\right]-\nabla \chi F\right)=\int d^{2} x \chi(x) \operatorname{Im}\left[\partial_{t} \bar{\psi}_{\sigma} \xi\right](x)
\end{aligned}
$$

hence

$$
\partial_{t}\left\langle G_{\delta(x)}^{\sigma}, \zeta\right\rangle=\operatorname{Im}\left[\partial_{t} \bar{\psi}_{\sigma}(x) \xi(x)\right] .
$$

Now, $K_{\sigma}^{-1}$ are uniformly bounded on $M_{a s}$ as operators from $H^{-1}$ to $H^{1}$ (see [ST1]) and we can estimate

$$
\begin{aligned}
\mid \int d^{2} x & \int d^{2} y \overline{\partial_{t}\left\langle G_{\delta(x)}^{\sigma}, \zeta\right\rangle} K_{\sigma}^{-1}(x, y) \partial_{t}\left\langle G_{\delta(y)}^{\sigma}, \zeta\right\rangle|=|\left(\partial_{t}\left\langle G_{\delta(x)}^{\sigma}, \zeta\right\rangle, K_{\sigma}^{-1} \partial_{t}\left\langle G_{\delta(x)}^{\sigma}, \zeta\right\rangle\right) \mid \\
& \left.\leq\left\|\partial_{t}\left\langle G_{\delta(x)}^{\sigma}, \zeta\right\rangle\right\|_{H^{-1}} \| K_{\sigma}^{-1} \partial_{t}\left\langle G_{\delta(x)}^{\sigma}, \zeta\right\rangle\right) \|_{H^{1}} \\
& \leq\left\|K_{\sigma}^{-1}\right\|_{H^{-1} \rightarrow H^{1}}\left\|\partial_{t}\left\langle G_{\delta(x)}^{\sigma}, \zeta\right\rangle\right\|_{H^{-1}}^{2}=C\left\|\operatorname{Im}\left[\partial_{t} \bar{\psi}_{\sigma} \xi\right]\right\|_{H^{-1}}^{2} \leq C\left\|\partial_{t} \psi_{\sigma} \xi\right\|_{H^{-1}}^{2}
\end{aligned}
$$


were here again $\zeta=(\xi, F)$. We want to estimate the r.h.s. of this inequality. We have

$$
\begin{aligned}
\left\|\partial_{t} \bar{\psi}_{\sigma} \xi\right\|_{H^{-1}} & =\sup _{\|\alpha\|_{H^{1}} \leq 1}\left\langle\alpha, \partial_{t} \bar{\psi}_{\sigma} \xi\right\rangle \leq \sup _{\|\alpha\|_{H^{1}} \leq 1}\|\alpha \xi\|_{2}\left\|\partial_{t} \psi_{\sigma}\right\|_{2} \\
& \leq \sup _{\|\alpha\|_{H^{1}} \leq 1}\|\alpha\|_{4}\left\|\partial_{t} \psi_{\sigma}\right\|_{2}\|\xi\|_{4}=C\left\|\partial_{t} \psi_{\sigma}\right\|_{2}\|\xi\|_{H_{v_{\sigma}}^{1}} .
\end{aligned}
$$

Furthermore, if we use Eq. (3.13) to write explicitly the time derivative

$$
\partial_{t} \psi_{\sigma}=i \dot{\tilde{\gamma}} \psi_{\sigma}+\sum_{i}\left(-\dot{z}_{i}\right)\left(\nabla_{A_{\sigma}} \psi_{\sigma}\right)_{i}
$$

we obtain the following bound on $\left\|\partial_{t} \psi_{\sigma}\right\|_{2}$

$$
\left\|\partial_{t} \psi_{\sigma}\right\|_{2} \leq\|\dot{\tilde{\gamma}}\|_{2}+\sum_{i}\left|\dot{z}_{i}\right|\left\|\left(\nabla_{A_{\sigma}} \psi_{\sigma}\right)_{i}\right\|_{2} \leq C\left(\|\dot{\tilde{\gamma}}\|_{2}+\sum_{i}\left|\dot{z}_{i}\right|\right)
$$

where again we use the gauge invariance of $\left\|\left(\nabla_{A_{\sigma}} \psi_{\sigma}\right)_{i}\right\|_{2}$.

Thus we arrive at the result

$$
\left\|\dot{P}_{v_{\sigma}} \zeta\right\|_{2} \leq C\left(\|\dot{\tilde{\gamma}}\|_{2}+\sum_{i}\left|\dot{z}_{i}\right|\right)\|\zeta\|_{H_{v_{\sigma}}^{1}}
$$

This completes the proof of Lemma C.

\section{Proof of Lemma D:}

Our goal is to prove the inequality

$$
\left|a_{n} \dot{\mathbf{z}}+\partial_{\mathbf{z}}^{A} \mathcal{E}_{\epsilon}^{\prime}\left(v_{\sigma}\right)\right|+\|\dot{\tilde{\gamma}}\|_{H^{1-s}} \leq C\left\|\dot{v}_{\sigma}+P_{v_{\sigma}} \mathcal{E}_{\epsilon}^{\prime}\left(v_{\sigma}\right)\right\|_{H_{v_{\sigma}}^{-s}}
$$

Starting with $\dot{\tilde{\gamma}}$ we have

$$
\begin{aligned}
\left\langle G_{\delta(x)}^{\sigma}, \dot{v}_{\sigma}+P_{v_{\sigma}} \mathcal{E}_{\epsilon}^{\prime}\left(v_{\sigma}\right)\right\rangle & =\left\langle G_{\delta(x)}^{\sigma}, \dot{v}_{\sigma}\right\rangle+\left\langle G_{\delta(x)}^{\sigma}, P_{v_{\sigma}} \mathcal{E}_{\epsilon}^{\prime}\left(v_{\sigma}\right)\right\rangle=\left\langle G_{\delta(x)}^{\sigma}, \dot{v}_{\sigma}\right\rangle \\
& =\left(-\Delta+\left|\psi_{\sigma}(x)\right|^{2}\right) \dot{\tilde{\gamma}}(x)=\left(K_{\sigma} \dot{\tilde{\gamma}}\right)(x)
\end{aligned}
$$

since $\left\langle G_{\delta(x)}^{\mathbf{z} \gamma}, P_{v} \mathcal{E}_{\epsilon}^{\prime}(v)\right\rangle=\operatorname{Im}[\bar{\psi}(\epsilon W(x) \psi)]=0$. We find the following expression for $\dot{\tilde{\gamma}}$

$$
\dot{\tilde{\gamma}}(x)=\int d^{2} y K_{\sigma}^{-1}(x, y)\left\langle G_{\delta(y)}^{\sigma}, \dot{v}_{\sigma}+P_{v_{\sigma}} \mathcal{E}_{\epsilon}^{\prime}\left(v_{\sigma}\right)\right\rangle
$$

where,

$$
\left\langle G_{\delta(y)}^{\sigma}, \dot{v}_{\sigma}+P_{v_{\sigma}} \mathcal{E}_{\epsilon}^{\prime}\left(v_{\sigma}\right)\right\rangle=\left\{\operatorname{Im}\left[\bar{\psi}_{\sigma}\left(\dot{v}_{\sigma}+P_{v_{\sigma}} \mathcal{E}_{\epsilon}^{\prime}\left(v_{\sigma}\right)\right)_{1}\right]-\nabla \cdot\left(\dot{v}_{\sigma}+P_{v_{\sigma}} \mathcal{E}_{\epsilon}^{\prime}\left(v_{\sigma}\right)\right)_{2}\right\}(y)
$$

$\operatorname{Here}\left(\dot{v}_{\sigma}+P_{v_{\sigma}} \mathcal{E}_{\epsilon}^{\prime}\left(v_{\sigma}\right)\right)_{1}$ is the first, complex scalar, component and $\left(\dot{v}_{\sigma}+P_{v_{\sigma}} \mathcal{E}_{\epsilon}^{\prime}\left(v_{\sigma}\right)\right)_{2}$ is the second, real vector component. Since $K_{\sigma}^{-1}$ is a bounded operator from $H^{-1-s}$ to $H^{1-s}$ (uniformly in $\sigma$ ) we have 


$$
\|\dot{\tilde{\gamma}}\|_{H^{1-s}}=\left\|K_{\sigma}^{-1}\left\langle G_{\delta(y)}^{\sigma}, \dot{v}_{\sigma}+P_{v_{\sigma}} \mathcal{E}_{\epsilon}^{\prime}\left(v_{\sigma}\right)\right\rangle\right\|_{H^{1-s}} \leq C\left\|\left\langle G_{\delta(y)}^{\sigma}, \dot{v}_{\sigma}+P_{v_{\sigma}} \mathcal{E}_{\epsilon}^{\prime}\left(v_{\sigma}\right)\right\rangle\right\|_{H^{-1-s}}
$$

However, using Eq. (5.18) we obtain

$$
\begin{aligned}
\|\left\langle G_{\delta(y)}^{\sigma}, \dot{v}_{\sigma}\right. & \left.+P_{v_{\sigma}} \mathcal{E}_{\epsilon}^{\prime}\left(v_{\sigma}\right)\right\rangle \|_{H^{-1-s}}= \\
& =\left\|\operatorname{Im}\left[\bar{\psi}_{\mathbf{z} 0} e^{-i \gamma}\left(\dot{v}_{\sigma}+P_{v_{\sigma}} \mathcal{E}_{\epsilon}^{\prime}\left(v_{\sigma}\right)\right)_{1}\right]-\nabla \cdot\left(\dot{v}_{\sigma}+P_{v_{\sigma}} \mathcal{E}_{\epsilon}^{\prime}\left(v_{\sigma}\right)\right)_{2}\right\|_{H^{-1-s}} \\
& \leq C\left\|\dot{v}_{\sigma}+P_{v_{\sigma}} \mathcal{E}_{\epsilon}^{\prime}\left(v_{\sigma}\right)\right\|_{H_{v_{\sigma}}^{-s}}
\end{aligned}
$$

which is the desired bound. As for $\dot{z}_{i}$, we have

$$
\left|a_{n} \dot{z}_{i}+\partial_{z_{i}}^{A} \mathcal{E}_{\epsilon}\left(v_{\sigma}\right)\right|=\left|\left\langle T_{i}^{\sigma}, \dot{v}_{\sigma}+\mathcal{E}_{\epsilon}^{\prime}\left(v_{\sigma}\right)\right\rangle\right| \leq\left\|T_{i}^{\sigma}\right\|_{H_{v_{\sigma}}^{s}}\left\|\dot{v}_{\sigma}+P_{v_{\sigma}} \mathcal{E}_{\epsilon}^{\prime}\left(v_{\sigma}\right)\right\|_{H_{v_{\sigma}}^{-s}} .
$$

Obviously $\left\|T_{i}^{\sigma}\right\|_{H_{v \sigma}^{s}}=\left\|T_{i}^{\mathbf{z} 0}\right\|_{H^{s}}<C$. From the last estimate, together with Eq. (5.19) and Eq. (5.20), follows Eq. (5.15).

\subsection{Proof of auxiliary technical lemmas Proof of Lemma A:}

For any $v \in M_{a s}$, the tangent space $T_{v} M_{a s}$ is spanned by zero eigenvectors of $L_{0 ; v}$. Hence, since $P_{v}$ is a projection on $T_{v} M_{a s}$ we have

$$
P_{v} L_{0 ; v}=L_{0 ; v} P_{v}=0 .
$$

Furthermore, by assumption the potential $W$ is bounded

$$
\sup _{x \in R^{2}}|W(x)|=C .
$$

Take any vector $\zeta=(\xi, F) \in T_{v} X^{(n)}$. The above observation leads to the following estimate

$$
\left\|P_{v} L_{\epsilon ; v} \zeta\right\|_{2}=\left\|P_{v}\left(L_{0 ; v}+\epsilon W\right) \zeta\right\|_{2} \leq \epsilon\|W \zeta\|_{2} \leq \epsilon C\|\zeta\|_{2} .
$$

\section{Proof of Lemma B:}

We set out to prove the following inequality

$$
\left\|P_{v} N_{v}(\zeta)\right\|_{H_{v_{\sigma}}^{-s}} \leq C\left[\|\zeta\|_{H_{v_{\sigma}}^{1}}^{2}+\|\zeta\|_{H_{v_{\sigma}}^{1}}^{3}\right] .
$$

The expression for $N_{v}(\zeta)$ is given in Appendix A, Eq. (A.3), where $\zeta=(\xi, F)$. The estimates of the terms in $N_{v}(\zeta)$ which do not include derivatives are straightforward. For example, denoting $\tilde{N}_{1}=(0,2 \operatorname{Re}(\bar{\psi} F \xi))$ we obtain 


$$
\left\|\tilde{N}_{1}\right\|_{H_{v_{\sigma}}^{-s}}=2\|\operatorname{Re}(\bar{\psi} F \xi)\|_{H_{v_{\sigma}}^{-s}} \leq 4\|\operatorname{Re}(\bar{\psi} F \xi)\|_{L_{v_{\sigma}}^{2}}=4\|\operatorname{Re}(\bar{\psi} F \xi)\|_{2} \leq C\|\zeta\|_{4}^{2} \leq C\|\zeta\|_{H_{v_{\sigma}}^{1}}^{2}
$$

The vector $\tilde{N}_{2}=\left(0, \operatorname{Im}\left(\bar{\xi} \nabla_{A} \xi\right)\right)$ is an example for a term which is more difficult to estimate. An estimate of this term is given in Subsection 3.2. The other problematic terms in $N_{v}(\zeta)$ are estimated in the same manner.

\section{Proof of Lemma E:}

We want to prove the inequality

$$
\left\|N_{v}(\zeta)\right\|_{2} \leq C\|\zeta\|_{H_{v_{\sigma}}^{2}}\left(\|\zeta\|_{H_{v_{\sigma}}^{1}}+\|\zeta\|_{H_{v_{\sigma}}^{1}}^{2}\right)
$$

Again the terms not containing any derivatives do not present any problems (see for example Eq. (5.26)). For the vector $\tilde{N}_{2}=\left(0, \operatorname{Im}\left(\bar{\xi} \nabla_{A_{\sigma}} \xi\right)\right)$ we have

$$
\begin{aligned}
\left\|\tilde{N}_{2}\right\|_{2} & \leq\left\|\bar{\xi} \nabla_{A_{\sigma}} \xi\right\|_{2} \leq\left\|\overline{\xi^{\prime}} \nabla_{A^{(n)}} \xi^{\prime}\right\|_{2} \leq\left\|\xi^{\prime}\right\|_{\infty}\left\|\nabla_{A^{(n)}} \xi^{\prime}\right\|_{2} \\
& \leq C\left\|\xi^{\prime}\right\|_{H^{2}}\left\|\xi^{\prime}\right\|_{H^{1}}=C\|\xi\|_{H_{v_{\sigma}}^{2}}\|\xi\|_{H_{v_{\sigma}}^{1}}
\end{aligned}
$$

where $\xi^{\prime}=e^{-i \gamma} \xi$. Other terms containing derivatives are estimated in a similar way.

\section{Proof of Lemma F:}

We want to prove the inequalities

$$
\left\|\bar{P}_{v_{\sigma}} L_{\epsilon ; v_{\sigma}} \zeta\right\|_{2} \leq C\|\zeta\|_{H_{v_{\sigma}}^{2}}
$$

and

$$
\left\|\bar{P}_{v_{\sigma}} L_{\epsilon ; v_{\sigma}} \zeta\right\|_{H_{v_{\sigma}}^{-1}} \leq C\|\zeta\|_{H_{v_{\sigma}}^{1}}
$$

Since $L_{\epsilon ; v_{\sigma}}$ is a second order linear operator no difficulties arise and the two inequalities are proved by strightforward calculations. We just note that

$$
\left\|\bar{P}_{v_{\sigma}} L_{\epsilon ; v_{\sigma}} \zeta\right\|_{2}=\left\|G_{-\gamma} \bar{P}_{v_{\sigma}} L_{\epsilon ; v_{\sigma}} \zeta\right\|_{2}=\left\|\bar{P}_{v_{\mathbf{z} 0}} L_{\epsilon ; v_{\mathbf{z} 0}} \zeta^{\prime}\right\|_{2}
$$

and

$$
\left\|\bar{P}_{v_{\sigma}} L_{\epsilon ; v_{\sigma}} \zeta\right\|_{H_{v_{\sigma}}^{-1}}=\left\|G_{-\gamma} \bar{P}_{v_{\sigma}} L_{\epsilon ; v_{\sigma}} \zeta\right\|_{H^{-1}}=\left\|\bar{P}_{v_{\mathbf{z} 0}} L_{\epsilon ; v_{\mathbf{z} 0}} \zeta^{\prime}\right\|_{H^{-1}}
$$

where $\zeta^{\prime}=e^{-i \gamma} \zeta$. Since the gauge function $\gamma$ does not appear in the operators on the r.h.s. of Eq. (5.31) and Eq. (5.32) one obtains by a straightforward calculation

$$
\left\|\bar{P}_{v_{\mathbf{z} 0}} L_{\epsilon ; v_{\mathbf{z} 0}} \zeta^{\prime}\right\|_{2} \leq C\left\|\zeta^{\prime}\right\|_{H^{2}}=\|\zeta\|_{H_{v_{\sigma}}^{2}}
$$

and

$$
\left\|\bar{P}_{v_{\mathbf{z} 0}} L_{\epsilon ; v_{\mathbf{z} 0}} \zeta^{\prime}\right\|_{H^{-1}} \leq C\left\|\zeta^{\prime}\right\|_{H^{1}}=C\left\|\zeta^{\prime}\right\|_{H_{v_{\sigma}}^{1}} .
$$




\section{Proof of Lemma G:}

We want to prove the inequality

$$
\left\|\partial_{v_{\sigma}} R_{v_{\sigma}}(\zeta)\right\|_{H_{v_{\sigma}}^{1}} \leq\|\zeta\|_{H_{v_{\sigma}}^{2}}^{2}\|\zeta\|_{H_{v_{\sigma}}^{1}}
$$

By derivation of the expression for $R_{v}(\zeta)$ (Eq. (A.5)) we obtain, for $\zeta=(\xi, F)$

$$
\partial_{v} R_{v}(\zeta)=\left(\left(F^{2}+\frac{\lambda}{2}|\xi|^{2}\right) \xi, F|\xi|^{2}\right)
$$

We want to estimate the norm $\left\|\partial_{v_{\sigma}} R_{v_{\sigma}}(\zeta)\right\|_{H_{v_{\sigma}}^{1}}$. Note first that

$$
G_{-\gamma} \partial_{v_{\sigma}} R_{v_{\sigma}}(\zeta)=\left(\left(F^{2}+\frac{\lambda}{2}\left|\xi^{\prime}\right|^{2}\right) \xi^{\prime}, F\left|\xi^{\prime}\right|^{2}\right)=\partial_{v_{\sigma}} R_{v_{\sigma}}\left(\zeta^{\prime}\right)
$$

where $\xi^{\prime}=e^{-i \gamma} \xi, \zeta^{\prime}=G_{-\gamma} \zeta$. The estimate of the $L^{2}$ norm (or $L_{v_{\sigma}}^{2}$ norm which is the same) is straightforward and we get

$$
\left\|\partial_{v_{\sigma}} R_{v_{\sigma}}(\zeta)\right\|_{2}^{2}=\left\|\left(F^{2}+\frac{\lambda}{2}\left|\xi^{\prime}\right|^{2}\right) \xi^{\prime}\right\|_{2}^{2}+\left\|F\left|\xi^{\prime}\right|^{2}\right\|_{2}^{2} \leq C\left\|\zeta^{\prime}\right\|_{H^{1}}^{6}=C\|\zeta\|_{H_{v_{\sigma}}^{1}}^{6}
$$

Let $\tilde{F}=F_{1}+i F_{2}$ be the complexification of $F$. We have

$$
\begin{aligned}
\left\|\nabla\left(\partial_{v} R_{v}\left(\zeta^{\prime}\right)\right)\right\|_{2}^{2} & =\left\|\nabla\left(\left(|\tilde{F}|^{2}+\frac{\lambda}{2}\left|\xi^{\prime}\right|^{2}\right) \xi^{\prime}\right)\right\|_{2}^{2}+\left\|\nabla\left(\tilde{F}\left|\xi^{\prime}\right|^{2}\right)\right\|_{2}^{2}= \\
& =\left\|2 \operatorname{Re}[(\nabla \tilde{F}) \overline{\tilde{F}}] \xi^{\prime}+|\tilde{F}|^{2}\left(\nabla \xi^{\prime}\right)+\lambda\left(\nabla \xi^{\prime}\right)\left|\xi^{\prime}\right|^{2}+\frac{\lambda}{2}\left(\nabla \overline{\xi^{\prime}}\right) \xi^{\prime 2}\right\|_{2}^{2} \\
& +\left\|(\nabla \tilde{F})\left|\xi^{\prime}\right|^{2}+2 \tilde{F} \operatorname{Re}\left[\overline{\xi^{\prime}} \nabla \xi^{\prime}\right]\right\|_{2}^{2} .
\end{aligned}
$$

All the terms on the r.h.s. of Eq. (5.34) are handled in the same manner. For example:

$$
\begin{aligned}
\left.\|(\nabla \tilde{F}) \overline{\tilde{F}} \xi^{\prime}\right) \|_{2} & \leq\|\tilde{F}\|_{\infty}\left\|\xi^{\prime}\right\|_{\infty}\|\nabla \tilde{F}\|_{2} \\
& \leq\|\tilde{F}\|_{H^{2}}\left\|\xi^{\prime}\right\|_{H^{2}}\|\tilde{F}\|_{H^{1}} \leq\left\|\zeta^{\prime}\right\|_{H^{2}}^{2}\left\|\zeta^{\prime}\right\|_{H^{1}}=\|\zeta\|_{H_{v_{\sigma}}^{2}}^{2}\|\zeta\|_{H_{v_{\sigma}}^{1}}
\end{aligned}
$$

\section{Proof of Lemma H:}

First we use Eq. (5.22) and the linear stability theorem in order to arrive at the result that for any $\zeta_{v} \in T_{v} X^{(n)}$ we have

$$
\begin{aligned}
\left\|L_{0 ; v} \zeta_{v}\right\|_{2}^{2} & =\left\langle L_{0 ; v} \zeta_{v}, L_{0 ; v} \zeta_{v}\right\rangle=\left\langle L_{0 ; v}^{1 / 2} \zeta_{v}, L_{0 ; v} L_{0 ; v}^{1 / 2} \zeta_{v}\right\rangle=\left\langle L_{0 ; v}^{1 / 2} \zeta_{v}, \bar{P}_{v} L_{0 ; v} \bar{P}_{v} L_{0 ; v}^{1 / 2} \zeta_{v}\right\rangle \\
& =\left\langle\bar{P}_{v} L_{0 ; v}^{1 / 2} \zeta_{v}, L_{0 ; v} \bar{P}_{v} L_{0 ; v}^{1 / 2} \zeta_{v}\right\rangle \geq \nu\left\|\bar{P}_{v} L_{0 ; v}^{1 / 2} \zeta_{v}\right\|_{2}^{2} .
\end{aligned}
$$


In addition, using Eq. (5.22) again, we have

$$
\left\|P_{v} L_{0 ; v}^{1 / 2} \zeta_{v}\right\|_{2}^{2}=\left\langle P_{v} L_{0 ; v} L_{0 ; v}^{-1 / 2} \zeta_{v}, P_{v} L_{0 ; v}^{1 / 2} \zeta_{v}\right\rangle=0 .
$$

From Eq. (5.35), Eq. (5.36) and the linear stability theorem we deduce that

$$
\left\langle L_{0 ; v} \zeta_{v}, L_{0 ; v} \zeta_{v}\right\rangle \geq \nu\left\langle\zeta_{v}, L_{0 ; v} \zeta_{v}\right\rangle \geq \nu^{2}\left\|\zeta_{v}\right\|_{H^{1}}^{2}
$$

We note that $\left\|\left(L_{0 ; v}+\Delta\right) \zeta_{v}\right\|_{2} \leq C_{1}\left\|\zeta_{v}\right\|_{H^{1}}+\left\|\nabla \nabla F_{v}\right\|_{2}$, where $\zeta_{v}=\left(\xi_{v}, F_{v}\right)$. Furthermore, since $\zeta_{v} \in\left(T_{v} M_{a s}\right)^{\perp}$, it satisfies the gauge condition $\operatorname{Im}\left(\bar{\psi}_{v} \xi_{v}\right)-\nabla F_{v}=0$. We conclude that $L_{0, v}+\Delta$ is a bounded operator from $H^{1}$ to $L^{2}$, i.e. $\left\|L_{0, v}+\Delta\right\|_{H^{1} \rightarrow L^{2}} \leq C_{1}$. we have

$$
\|\Delta \zeta\|_{2}^{2}=\left\|\left(L_{0 ; v}+\Delta\right) \zeta-L_{0 ; v} \zeta\right\|_{2}^{2} \leq 2\left(\left\|\left(L_{0 ; v}+\Delta\right) \zeta\right\|_{2}^{2}+\left\|L_{0 ; v} \zeta\right\|_{2}^{2}\right) \leq 2\left(C_{1}\|\zeta\|_{H^{1}}^{2}+\left\|L_{0 ; v} \zeta\right\|_{2}^{2}\right)
$$

and so

$$
\frac{1}{2}\|\Delta \zeta\|_{2}^{2}-C_{1}\|\zeta\|_{H^{1}}^{2} \leq\left\langle L_{0 ; v} \zeta, L_{0 ; v} \zeta\right\rangle
$$

Then, for any $0<\delta<1$ we have

$$
\begin{aligned}
\left\langle L_{0, v} \zeta_{v}, L_{0, v} \zeta_{v}\right\rangle & =\delta\left\langle L_{0, v} \zeta_{v}, L_{0, v} \zeta_{v}\right\rangle+(1-\delta)\left\langle L_{0, v} \zeta_{v}, L_{0, v} \zeta_{v}\right\rangle \\
& \geq \frac{\delta}{2}\left\|\Delta \zeta_{v}\right\|_{2}^{2}+\left[(1-\delta) C_{2}-\delta C_{1}\right]\left\|\zeta_{v}\right\|_{H^{1}}^{2}
\end{aligned}
$$

Choosing $\delta$ small enough the second term on the r.h.s. of Eq. (5.38) is positive and we get

$$
\left\langle L_{0, v} \zeta_{v}, L_{0, v} \zeta_{v}\right\rangle \geq C\left\|\zeta_{v}\right\|_{H^{2}}^{2} .
$$

In addition to this basic result we have

$$
\left\|L_{0 ; v} \zeta_{v}\right\|_{2}^{2}=\left\|\bar{P}_{v} L_{0 ; v} \zeta_{v}\right\|_{2}^{2}=\left\|\bar{P}_{v}\left(L_{\epsilon ; v} \zeta_{v}-\epsilon W \zeta_{v}\right)\right\|_{2}^{2} \leq 2\left(\left\|\bar{P}_{v} L_{\epsilon ; v} \zeta_{v}\right\|_{2}^{2}+\epsilon^{2}\|W\|_{\infty}^{2}\left\|\zeta_{v}\right\|_{2}^{2}\right)
$$

which leads to

$$
\left\langle\bar{P}_{v} L_{\epsilon ; v} \zeta_{v}, \bar{P}_{v} L_{\epsilon ; v} \zeta_{v}\right\rangle \geq\left(\frac{1}{2}-\epsilon^{2}\|W\|_{\infty}^{2} \nu^{2}\right)\left\langle L_{0 ; v} \zeta, L_{0 ; v} \zeta_{v}\right\rangle .
$$

The proof of Lemma $\mathrm{H}$ is completed by the following simple observation

$$
\left\langle\bar{P}_{v} L_{\epsilon ; v} \zeta_{v}, \bar{P}_{v} L_{\epsilon ; v} \zeta_{v}\right\rangle=\left\langle G_{-\gamma} \bar{P}_{v} L_{\epsilon ; v} \zeta_{v}, G_{-\gamma} \bar{P}_{v} L_{\epsilon ; v} \zeta_{v}\right\rangle=\left\langle\bar{P}_{v_{\mathbf{z} 0}} L_{\epsilon ; v_{\mathbf{z} 0}} \zeta_{v}^{\prime}, \bar{P}_{v_{\mathbf{z} 0}} L_{\epsilon ; v_{\mathbf{z} 0}} \zeta_{v}^{\prime}\right\rangle
$$

where $\zeta_{v}^{\prime}=G_{-\gamma} \zeta_{v}$ and we have $P_{v_{\mathbf{z} 0}} \zeta_{v}^{\prime}=0$. Eq. (5.39), Eq. (5.41) and Eq. (5.42) imply the desired result.

\section{Proof of Lemma I:}


The inequality in Lemma I, Eq. (4.23), follows from Eq. (5.37), Eq. (5.41) and the following simple estimate

$$
\begin{aligned}
\left\langle\zeta_{v}, L_{\epsilon ; v} \zeta_{v}\right\rangle & =\left\langle\zeta_{v}, L_{0 ; v} \zeta_{v}\right\rangle+\epsilon\left\langle\zeta_{v}, W \zeta_{v}\right\rangle \leq\left\langle\zeta_{v}, L_{0 ; v} \zeta_{v}\right\rangle+\epsilon\|W\|_{\infty}\left\|\zeta_{v}\right\|_{2}^{2} \\
& \leq\left(1+\epsilon\|W\|_{\infty} \nu\right)\left\langle\zeta_{v}, L_{0 ; v} \zeta_{v}\right\rangle .
\end{aligned}
$$

\section{Appendix A - Taylor expansions of $\mathcal{E}_{\epsilon}(u)$ and $\mathcal{E}_{\epsilon}^{\prime}(u)$ :}

In the proof of Theorem A above we frequently refer to the Taylor expansion of $\mathcal{E}_{\epsilon}^{\prime}(u)$

$$
\mathcal{E}_{\epsilon}^{\prime}(u)=\mathcal{E}_{\epsilon}^{\prime}(v)+L_{\epsilon ; v} \zeta+N_{\epsilon ; v}(\zeta)
$$

A strightforward calculation provides us with explicit expressions for the linear and nonlinear terms in $\zeta$. Denoting $\zeta=(\xi, F)$, we have

$$
L_{\epsilon ; u} \zeta=\left(\begin{array}{c}
{\left[-\Delta_{A}+\frac{\lambda}{2}\left(2|\psi|^{2}-1\right)\right] \xi+\frac{\lambda}{2} \psi^{2} \bar{\xi}+i\left[2 \nabla_{A} \psi+\psi \nabla\right] F+\epsilon W \xi} \\
\operatorname{Im}\left(\left[\overline{\nabla_{A}} \psi-\bar{\psi} \nabla_{A}\right] \xi\right)+\left(-\Delta+\nabla \nabla+|\psi|^{2}\right) F
\end{array}\right)
$$

and

$$
N_{v}(\zeta)=N_{\psi, A}(\zeta)=\left(\begin{array}{c}
i\left(2 \nabla_{A} \xi+\xi \nabla\right) F+F^{2} \psi+F^{2} \xi+\frac{\lambda}{2}\left(\bar{\psi} \xi^{2}+2|\xi|^{2} \psi+|\xi|^{2} \xi\right) \\
2 \operatorname{Re}(\bar{\psi} F \xi)-\operatorname{Im}\left(\bar{\xi} \nabla_{A} \xi\right)+F|\xi|^{2}
\end{array}\right)
$$

In addition we refer at several points to the Taylor expansion of the energy functional $\mathcal{E}_{\epsilon}^{\prime}(u)$. Letting $u=v+\zeta$ we have

$$
\mathcal{E}_{\epsilon}(u)=\mathcal{E}_{\epsilon}(v)+\left\langle\zeta, \mathcal{E}_{\epsilon}^{\prime}(v)\right\rangle+\frac{1}{2}\left\langle\zeta, L_{\epsilon ; v} \zeta\right\rangle+R_{v}(\zeta)
$$

where $L_{\epsilon ; v}$ is given in Eq. (A.2) and the remainder $R_{v}(\zeta)$ (containing all terms which are higher then quadratic in $\zeta$ ) is given by

$$
R_{v}(\zeta)=\int\left\{\left(F^{2}+\frac{\lambda}{2}|\xi|^{2}\right) \operatorname{Re}[\bar{\xi} \psi]-F \cdot \operatorname{Im}\left[\bar{\xi} \nabla_{A} \xi\right]+\frac{1}{2}\left(F^{2}|\xi|^{2}+\frac{\lambda}{4}|\xi|^{4}\right)\right\} d^{2} x
$$

\section{Acknowledgments}

The work of Y. Strauss was supported by THE ISRAEL SCIENCE FOUNDATION (grant no. 188/02) and the Edmund Landau Center for Research in Mathematical Analysis and Related areas, sponsored by the Minerva Foundation (Germany). 


\section{References}

[A] Abrikosov A.A.: On the magnetic properties of superconductors of the second group. Soviet Physics JETP 5 1174-1182 (1957).

[ABP] Andre N. and Bauman P. and Phillips D.: Vortex pinning with bounded fields for the Ginzburg-Landau equation. Ann. Ins. Henri Poincaré Anal. Non Linéaire 20 no. 4 705-729 (2003).

[AS] Andre N. and Shafrir I.: Asymptotic behavior of minimizers for the Ginzburg-Landau functional with weight. Arch. Rational Mech. Anal. 142, no. 1, 45-73 75-98 (1998).

[ASaSe] Aftalion A. and Sandier E. and Serfaty S.: Pinning phenomena in the GinzburgLandau model of superconductivity. J. Math. Pures Appl. (9) 80 no. 3 339-372 (2001).

[AH] Atiyah M. and Hitchin N.: The Geometry and Dynamics of Magnetic Monopoles. Princeton University Press (1988).

[BBH] Bethuel F. and Brezis H. and Hélein F.: Ginzburg-Landau Vortices, Birkhäuser (1994).

[BC] Berger M.S. and Chen Y.Y.: Symmetric vortices for the Ginzburg-Landau equations of superconductivity, and the nonlinear desingularization phenomenon. J. Func. Anal. 82 259-295 (1989).

[BFGLV] Blatter G. and Feigeĺman M.V. and Geshkenbein V.B. et. al.: Vortices in high temprature superconductors. Rev. Modern Phys. 661125 (1994).

[BR] Bethuel F. and Rivière T.: Vortices for a variational problem related to superconductivity. Ann. Inst. Henri Pincaré, Anal. Non Linéaire 12 no. 3 243-303 (1995).

[DG] Du Q. and Gunzburger M.D.: A model for superconducting thin films having variable thickness. Phys. D 69 215-231 (1993).

[DS] Demoulini S. and Stuart D.: Gradient flow of the superconducting Ginzburg-Landau functional on the plane. Comm. Anal. Geom. 5 121-198 (1997).

[E] E W.: Dynamics of vortices in Ginzburg-Landau thories with applications to superconductivity. Physica D 77 383-404 (1994).

[GE] Gork'ov L.P. and Eliashberg G.M.: Generalization of the Ginzburg-Landau equations for non-stationary problems in the case of alloys with paramagnetic impurities. Soviet Physics JETP 27 no. 2 328-334 (1968).

[GS1] Gustafson S. and Sigal I.M.: The stability of magnetic vortices. Comm. Math. Phys. 212 257-275 (2000).

[GS2] Gustafson S. and Sigal I.M.: Effective dynamics of magnetic vortices. Preprint, arXiv:math.AP/0312438 (2003).

[Gu] Gustafson S.: Some mathematical problems in the Ginzburg-Landau theory of superconductivity. Ph.D. thesis, Univ. Toronto (1999).

[JS] Jerard R. and Soner M.: Dynamics of Ginzburg-Landau vortices. Arch. Rational Mech. Anal. 142 no. 2, 99-125 (1998).

[JT] Jaffe A. and Taubes C.: Vortices and monopoles. Structure of static gauge theories. Progr. in Phys. Vol. 2 Birkhäuser, Boston, MA 1980. 
[L] Lin F.H.: Some dynamical properties of Ginzburg-Landau vortices. Comm. Pure. Appl. Math. 49 no. 4 323-359 (1996).

[N] Neu J.: Vortices in complex scalar fields. Physica D 43 385-406 (1990).

[P] Plohr B. Ph.D. thesis, Princeton (1978).

[PaRi] Pacard F. and Rivière T.: Linear and Nonlinear Aspects of Vortices, Birkhäuser, Boston (2000).

[PeRu] Peres L. and Rubinstein J.: Vortex dynamics in $U(1)$ Ginzburg-Landau models. Physica D 64 299-309 (1993).

[Rit] Ritter W.G.: Gauge theory: instantons, monopoles and moduli spaces. Preprint, arXiv:math-ph/0304026 v1 (2003).

[Riv] Riviére T.: Ginzburg-Landau vortices: the static model. In Sémininaire Bourbaki, Vol. 1999/2000, Astérisque No. 276 73-103 (2002).

[Rub1] Rubinstein J.: On the equlibrium position of Ginzburg-Landau vortices. Z. Angew. Math. Phys. 46 no. 5 739-751 (1995).

[Rub2] Rubinstein J.: Six lectures on superconductivity. In boundaries, interfaces and Transitions (Banff. AB, 1995) CRM Proc. Lecture Notes, Vol. 13, Amer. Math. Soc., Providence, RI, pp. 163-184 (1998).

[RS] Rubinstein J. and Sternberg P.: On the slow motion of vortices in the GinzburgLandau heat flow. SIAM J. Math. Anal. 26 no. 6 1452-1466 (1995).

[S] Struwe M.: On the asymptotic behavior of minimizers of the Ginzburg-Landau model in 2-dimensions. J. Diff. Int. Equations 7 1613-1624 (1994) and Erratum in J. Diff. Int.

[ST1] Sigal I.M. and Ting F.: Pinning of magnetic vortices by an external potential. Russian (2004).

[ST2] Sigal I.M. and Ting F.: Stability of pinned vortices (to appear).

[T] Tinkham M.: Introduction to superconductivity. McGraw-Hill, New York (1975).

Department of Mathematics

University of Toronto

Toronto, ON, Canada, M5S 3G3

URL: www.math.toronto.edu/sigal

Department of Mathematics

University of Notre Dame

Notre Dame, IN, USA, 46556-4618

Einstein Institute of Mathematics

The Hubrew University of Jerusalem

Jerusalem, Israel 91904

e-mail: ystrauss@math.huji.ac.il 